\title{
Instability analysis of nonlinear surface waves in a circular cylindrical container subjected to a vertical excitation
}

\author{
Yongjun Jian ${ }^{\mathrm{a}, \mathrm{b}, *}$, Xuequan $\mathrm{E}^{\mathrm{a}}$ \\ a Institute of Mechanics, Chinese Academy of Sciences, Beijing 100080, China \\ ${ }^{\mathrm{b}}$ First Institute of Oceanography, State Oceanic Administration, Qingdao 266061, China \\ Received 10 May 2004; received in revised form 27 January 2005; accepted 7 March 2005
}

Available online 17 May 2005

\begin{abstract}
Singular perturbation theory of two-time scale expansions was developed both in inviscid and weak viscous fluids to investigate the motion of single surface standing wave in a liquid-filled circular cylindrical vessel, which is subject to a vertical periodical oscillation. Firstly, it is assumed that the fluid in the circular cylindrical vessel is inviscid, incompressible and the motion is irrotational, a nonlinear evolution equation of slowly varying complex amplitude, which incorporates cubic nonlinear term, external excitation and the influence of surface tension, was derived from solvability condition of high-order approximation. It shows that when forced frequency is low, the effect of surface tension on mode selection of surface wave is not important. However, when forced frequency is high, the influence of surface tension is significant, and can not be neglected. This proved that the surface tension has the function, which causes free surface returning to equilibrium location. Theoretical results much close to experimental results when the surface tension is considered. In fact, the damping will appear in actual physical system due to dissipation of viscosity of fluid. Based upon weakly viscous fluids assumption, the fluid field was divided into an outer potential flow region and an inner boundary layer region. A linear amplitude equation of slowly varying complex amplitude, which incorporates damping term and external excitation, was derived from linearized Navier-Stokes equation. The analytical expression of damping coefficient was determined and the relation between damping and other related parameters (such as viscosity, forced amplitude and depth of fluid) was presented. The nonlinear amplitude equation and a dispersion, which had been derived from the inviscid fluid approximation, were modified by adding linear damping. It was found that the modified results much reasonably close to experimental results. Moreover, the influence both of the surface tension and the weak viscosity on the mode formation was described by comparing theoretical and experimental results. The results show that when the forcing frequency is low, the viscosity of the fluid is prominent for the mode selection. However, when the forcing frequency is high, the surface tension of the fluid is prominent. Finally, instability of the surface wave is analyzed and properties of the solutions of the modified amplitude equation are determined together with phase-plane trajectories. A necessary condition of forming stable surface wave is obtained and unstable regions are illustrated.
\end{abstract}

(C) 2005 Elsevier SAS. All rights reserved.

\footnotetext{
* Corresponding author. Tel.: +86 030532 7105680; fax: +86 0305328967094 (Y. Jian).

E-mail addresses: jianyongjun@yahoo.com.cn (Y. Jian), exq@imech.ac.cn (X. E).
} 


\section{Introduction}

Nearly all of the liquid-filled containers with which we are familiar, ranging from the common teacup to the huge rocket propellant tanks, the common feature is that the contained liquid has free surface. When the container is subjected to external excitation, the surface waves occur at the free surface. There are many actual engineering application backgrounds associated with the motions of surface waves in the oscillating liquid-filled rigid vessel, such as liquid-filled spaceflight system, fuel tanks of missiles, other launch vehicles [1] and aseismatic problem of the large liquid tank [2]. These surface waves can impose force on the container, and affect stability of the aircraft. By the study of liquid dynamic behavior in moving containers, people can control the moving stability and provide theoretical guidance to systematic engineering design.

The experimental study of surface waves on a fluid via vertical periodic vibration in a closed cylindrical vessel was carried out by Faraday [3]. He found that these surface waves have a frequency equal to one half that of the excitation and belong to subharmonic resonance. Miles [4] designated those waves associated with an oscillation of the effective gravitational acceleration as Faraday waves. Non-propagating solitary waves are one kind of Faraday waves, and both of them describe the wave motion at the free surface in a vertical periodic oscillating vessel [5]. The principle discrepancy between non-propagating solitary wave and Faraday wave is that the former is formed in a long and narrow rectangular vessel, and the Faraday wave patterns are produced in a tank which has the same scale of length and width. Moreover, the study of Faraday wave is related to complex phenomenon, such as instability and spatio-temporal chaos [6,7]. It is noticeable that the internal solitary wave is a kind of new environmental load factor in the ocean engineering. The existence of large-scale internal soliton waves in the ocean has important influence on the safety navigation of fleet and submarine in military engineering. This kind of study has very strong practical background when the case of liquid vertical periodic motion is studied [8].

Until the 1950's, Benjamin and Ursell [9] proved theoretically that the linear dynamics of the amplitudes of the surface modes is governed by Mathieu's equation, and consolidated the dispute associated with this problem. Miles [10-12] has studied nonlinear effects, adopting a variational approach.

In the last twenty years, due to the further improvement of computer level and of flow visualization technique, Faraday instability problem continues to attract the attention of many researchers. In the recent years, the aim of many studies focused on nonlinear pattern-forming [13,14], bifurcation, chaos [15,16] and the transition from ordered patterns to spatio-temporal disorder $[17,18]$. Due to complex variability and richness of observational modes in Faraday system, many flowing patterns were found in experiments via single or multiple frequency vertical excitations, such as square patterns, two-dimensional quasicrystal structure, hexagons, triangles, parallel, spiral line and twelvefold quasi-periodic patterns, etc.

E and Gao [19-21] carried out the flow visualization and experimental study on surface wave patterns in a circular cylindrical vessel subjected to a vertical external vibration. They obtained very beautiful photographys of the free surface patterns in wide driven frequency, and most of them have not been reported before. Recently, Jian and E [22,23] proposed a mathematical formulation associated with the flowing visualization in Refs. [19-21], from which a nonlinear amplitude equation, the second order free surface displacements and their contours of the nonlinear Faraday waves were obtained by two-time scale singular perturbation expansion in inviscid fluid. Although the contours of free surface waves agreed well with the experimental visualization, the external vertical forced frequency has large difference.

In the aspect of viscous damping of surface wave, Ursell [24] developed an energy-dissipation method to describe the dissipation in the vicinity of vertical walls when the depth of the water is infinite. Mei and Liu [25] were the first to show how to correctly treat damping in closed containers without considering the effect of surface tension of the surface wave. Bernoff et al. [26] completely solved the first-order damping for a rectangular container. Case and Parkinson [27] studied the damping of surface waves in a cylindrical container. The analytical expressions of the damping were obtained and their theory and experiment were found to be in good agreement. Henderson and Miles [28] calculated the natural frequencies and damping ratios for surface waves in a circular cylinder on the assumptions of a fixed contact line. The predicted and observed frequencies agreed very well each other, but the difference between the predicted and observed damping ratio is large. Carlos Martel et al. [29] improved the difference of [28] by considering higher-order approximations that include the effect of viscous dissipation in the Stokes boundary layers and the bulk.

Surface tension has important effect on the small scale surface waves. Krasnopolskays and van Heijst [30] studied wave pattern formation in a fluid annulus with a radially vibrating inner cylinder both theoretically and experimentally, which includes the influence of surface tension. The experimental observations agree very well with the theoretical results. The influence of surface tension and viscosity of the fluid on the mode formation should be taken into account in Refs. [22,23]. The purpose of this paper is that the dispersive relation and nonlinear amplitude equation in Refs. [22,23] can be modified by adding the surface tension in inviscid fluid and the damping coefficient in weakly viscid fluid, which is obtained in theory other than via previous empirical method. Theoretical forced frequency is much closer to experimental result than that of no surface tension and damping for a same excited surface mode.

This paper is organized as follows. In Section 2, the effect of surface tension on vertical forced surface waves is presented. In Section 3, we formulate the vertical excited surface wave problem in a weakly viscous fluid. The asymptotic solutions of 
both external potential flow and internal boundary layer region are obtained by two-time scale asymptotic expansion, and linear damping coefficient is derived from amplitude equation. Moreover, a comparison between our theoretical damping and Case and Parkinson's results in Ref. [27] is made. In Section 4, the instability of the modified amplitude equation is discussed and unstable regions are determined by stability analysis with respect to the infinitesimal disturbance to the equilibrium solution. In Section 5, many numerical results, such as dispersive relations, variation of the damping coefficient, the characters of the surface waves, the regions of instability, phase-plane trajectory and evolution with time of amplitude equation, etc. are presented. Finally, in Section 6, the conclusions are presented.

\section{The theory of vertical forced surface waves with the effect of surface tension in inviscid fluid}

\subsection{Governing equations and boundary conditions}

We consider surface waves excited by the vertical motion of a circular cylindrical basin filled with inviscid fluid with the effect of surface tension, as shown in Fig. 1. We take cylindrical coordinate system $(r, \theta, z)$ moving with the vessel, such that the equation of the undisturbed free surface is $z=0$, and base of the vessel is located at $z=-h<0$. Here, $h$ denotes the depth of fluid. If the vessel motion is assumed to be at a vertical acceleration $\ddot{z}_{0}$, then the fluid moves relatively to the vessel as if it moves at a gravitational acceleration $\left(g-\ddot{z}_{0}\right)$ in a stationary vessel, where $z_{0}=A \cos \left(2 \omega_{0} t\right)$ is the excited displacement of the vessel in the vertical direction, $\ddot{z}_{0}$ is the derivative of second order with respect to time $t$, while $A$ and $2 \omega_{0}$ are the amplitude and angular frequency of the external forcing. It is assumed that the fluid is incompressible and the motion is irrotational, there must be exist a velocity potential function $\phi(r, \theta, z, t)$, which satisfies the following governing equation

$$
\frac{\partial^{2} \phi}{\partial r^{2}}+\frac{1}{r} \frac{\partial \phi}{\partial r}+\frac{1}{r^{2}} \frac{\partial^{2} \phi}{\partial \theta^{2}}+\frac{\partial^{2} \phi}{\partial z^{2}}=0, \quad 0<r<R,-h \leqslant z \leqslant \eta(r, \theta, t)
$$

with kinetic boundary condition

$$
\frac{\partial \phi}{\partial t}+\frac{1}{2}\left[\left(\frac{\partial \phi}{\partial r}\right)^{2}+\frac{1}{r^{2}}\left(\frac{\partial \phi}{\partial \theta}\right)^{2}+\left(\frac{\partial \phi}{\partial z}\right)^{2}\right]+\left(g-\ddot{z}_{0}\right) \eta-\frac{\Gamma}{\rho}\left(\frac{\partial^{2} \eta}{\partial r^{2}}+\frac{1}{r} \frac{\partial \eta}{\partial r}+\frac{1}{r^{2}} \frac{\partial^{2} \eta}{\partial \theta^{2}}\right)=0, \quad z=\eta(r, \theta, t)
$$

and kinematic condition

$$
\frac{\partial \eta}{\partial t}+\frac{1}{r^{2}} \frac{\partial \phi}{\partial \theta} \frac{\partial \eta}{\partial \theta}+\frac{\partial \phi}{\partial r} \frac{\partial \eta}{\partial r}-\frac{\partial \phi}{\partial z}=0, \quad z=\eta(r, \theta, t)
$$

at free surface. Where $\eta(r, \theta, t)$ is displacement of the surface from $z=0, R$ is the internal radius of the container, constants $\Gamma$ and $\rho$ denote the surface tension coefficient and the density of the fluid respectively. The effect of surface tension is introduced by boundary condition of the free surface and its high-order nonlinear terms are ignored in this paper. In addition, since the influence of viscosity is ignored, the boundary conditions on the side-wall and at the bottom of the vessel become zero normal velocity for rigid container, namely

$$
\begin{aligned}
& \frac{\partial \phi}{\partial r}=0, \quad r=R, \\
& \frac{\partial \phi}{\partial z}=0, \quad z=-h .
\end{aligned}
$$

Eqs. (2.1)-(2.5) establish the mathematical modeling that describes the nonlinear surface wave in inviscid fluid with the effect of the surface tension. Following we will solve these equations by the two-time scale perturbation expansion technique.

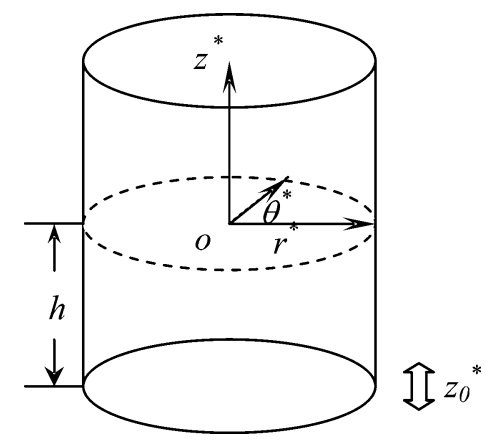

Fig. 1. Physical model of the liquid-filled circular cylinder. 


\subsection{The solution of the problem and derivation of amplitude equation}

Taking the radius $R$ of the vessel as the length scale, and nondimensionalizing all related independent and unknown variables, the following scalings are adopted

$$
\begin{aligned}
& z^{*}=z / R, \quad r^{*}=r / R, \quad \eta^{*}=\eta / R, \quad t^{*}=t / \sqrt{R / g}, \quad \phi^{*}=\phi /(R \sqrt{g R}), \quad \omega_{0}^{*}=\omega_{0} / \sqrt{g / R}, \\
& A^{*}=A / R, \quad \varepsilon^{* 2}=4 A \omega_{0}^{2} / g, \quad \Gamma^{*} / \rho^{*}=\left(g R^{2}\right) \Gamma / \rho .
\end{aligned}
$$

Note that the asterisks denote dimensionless quantities and are subsequently dropped. The parameter $\varepsilon^{*}$ quantifies the acceleration due to the vertical oscillation relative to gravity and is assumed to be much less than unit. Substituting (2.6) into (2.1)-(2.5), then expanding (2.2) and (2.3) into Taylor series at $z=0$ by neglecting of the term $\mathrm{O}\left(\varepsilon^{4}\right)$, nondimensional governing equation and boundary conditions can be obtained. In order to use the method of two-time scale perturbation expansion, a slowly varying time scale $\tau$ is introduced, and let $\tau=\varepsilon^{2} t$, we have

$$
\partial / \partial t=\partial / \partial t+\varepsilon^{2} \partial / \partial \tau+\cdots
$$

where $\partial / \partial t$ denotes the derivative about the time $t$.

The complexity of this problem is that the free surface is unknown, and must be solved together with velocity potential. To seek the solutions of $\phi(r, \theta, z, t)$ and $\eta(r, \theta, t)$, we expand them in a power series in $\varepsilon$ as

$$
\begin{aligned}
& \phi(r, \theta, z, t, \tau)=\varepsilon \phi_{1}+\varepsilon^{2} \phi_{2}+\varepsilon^{3} \phi_{3}+\cdots, \\
& \eta(r, \theta, t, \tau)=\varepsilon \eta_{1}+\varepsilon^{2} \eta_{2}+\varepsilon^{3} \eta_{3}+\cdots .
\end{aligned}
$$

Substituting (2.7) and (2.8) into nondimensional equations, we can give each order approximate equation by comparing the coefficients of the small parameter $\varepsilon^{i}$ at the two sides of the equations. The method used here is similar to that in Refs. [22,23], and the only difference between them is that the influence of surface tension appears in the free surface boundary condition. Due to the complexity of the mathematical formulation, the detailed deriving process is ignored, and only the important results are given in followings. The first order velocity potential $\phi_{1}(r, \theta, z, t, \tau)$ and free surface displacement $\eta_{1}(r, \theta, t, \tau)$ can be expressed as

$$
\begin{aligned}
& \phi_{1}=J_{m}(\lambda r) \cosh [\lambda(z+h / R)] \cdot\left[p(\tau) \mathrm{e}^{\mathrm{i} \Omega t}+\bar{p}(\tau) \mathrm{e}^{-\mathrm{i} \Omega t}\right] \cos m \theta, \\
& \eta_{1}=\frac{\lambda}{\mathrm{i} \Omega} J_{m}(\lambda r) \sinh (\lambda h / R) \cdot\left[p(\tau) \mathrm{e}^{\mathrm{i} \Omega t}-\bar{p}(\tau) \mathrm{e}^{-\mathrm{i} \Omega t}\right] \cos m \theta .
\end{aligned}
$$

The dispersion relationship is

$$
\Omega^{2}=\lambda_{m n} \tanh \left(\lambda_{m n} h / R\right)\left(1+\frac{\Gamma}{\rho} \lambda_{m n}^{2}\right)=\Omega_{m n}^{2} .
$$

Where $\lambda=\lambda_{m n}$ are the positive real roots of $\mathrm{d} J_{m}\left(\lambda_{m n} r\right) /\left.\mathrm{d} r\right|_{r=1}=0, J_{m}(r)$ is the $m$-th order Bessel function of first kind, $p(\tau)$ is called the slowly variable complex amplitude and $\bar{p}(\tau)$ denotes the complex conjugate of $p(\tau)$, and $\Omega$ is the natural frequency of the surface wave.

The second order velocity potential $\phi_{2}(r, \theta, z, t, \tau)$ and free surface displacement $\eta_{2}(r, \theta, t, \tau)$ can be expressed as

$$
\begin{aligned}
& \phi_{2}(r, \theta, z, t, \tau)=\left[X_{1}(r, z)+X_{2}(r, z) \cos (2 m \theta)\right] \cdot\left(p^{2}(\tau) \mathrm{e}^{2 \mathrm{i} \Omega t}-\bar{p}^{2}(\tau) \mathrm{e}^{-2 \mathrm{i} \Omega t}\right), \\
& \eta_{2}(r, \theta, t, \tau)=\left[Y_{1}(r)+Y_{2}(r) \cos (2 m \theta)\right] \cdot\left(p^{2}(\tau) \mathrm{e}^{2 \mathrm{i} \Omega t}+\bar{p}^{2}(\tau) \mathrm{e}^{-2 \mathrm{i} \Omega t}\right) .
\end{aligned}
$$

Where $Y_{1}(r)$ and $Y_{2}(r)$ are the functions of the variable $r$, and $X_{1}(r, z)$ and $X_{2}(r, z)$ are functions of the variable $r$ and $z$. The detail expressions of them are ignored in this paper.

We have solved the second order velocity potential and displacement of free surface equations. However, the slowly varying amplitude $p(\tau)$ is still not determined. We continue to consider the third order governing equations and boundary conditions, using the solvability condition of the third order problem to derive the amplitude equation of $p(\tau)$. Since third order problem are inhomogeneous and the first order homogeneous problem has a nontrivial solution, it is necessary to apply a solvability condition to ensure the availability of the third order problem. It is assumed that the frequency $\Omega$ of the free surface wave is close to one half the forced frequency $\omega_{0}$, and let $\omega_{0}-\Omega=\varepsilon^{2} \sigma$. Finally, the solvability condition is obtained

$$
\mathrm{i} \frac{\mathrm{d} p(\tau)}{\mathrm{d} \tau}=M_{1} p^{2}(\tau) \bar{p}(\tau)+M_{2} \mathrm{e}^{2 \mathrm{i} \sigma \tau} \bar{p}(\tau)
$$

where $\mathrm{i}$ is unit of imaginary number, $M_{1}$ and $M_{2}$ are constants. Although the form of (2.14) is equivalent to Eq. (65) in Ref. [22], the former includes the effect of surface tension in the coefficients of $M_{1}$ and $M_{2}$. We define $p_{1}(\tau)$ and $p_{2}(\tau)$ as the real and 


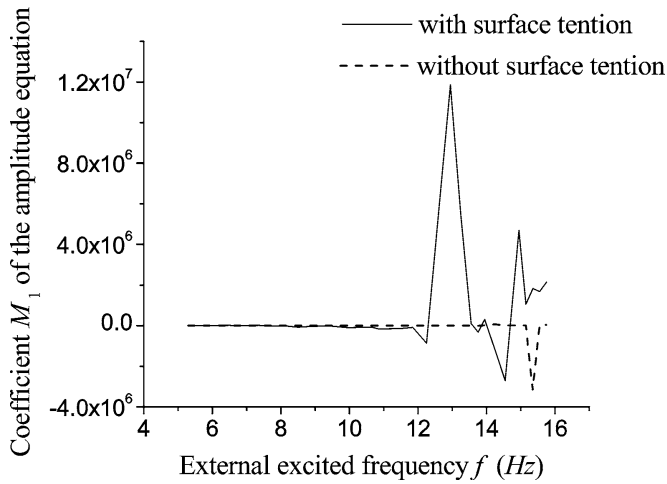

(a)

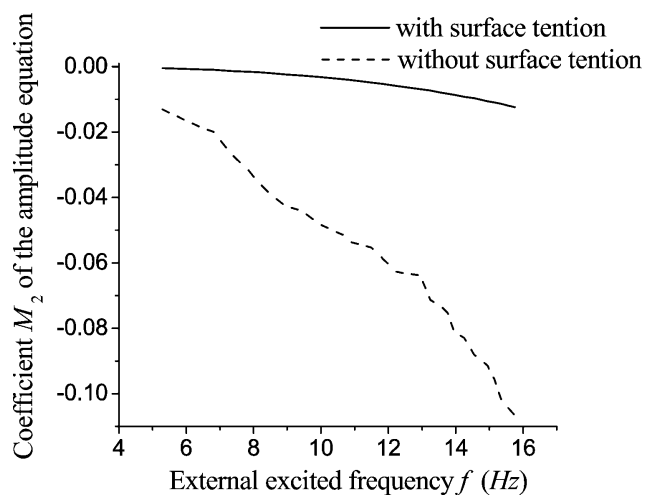

(b)

Fig. 2. Variation of the coefficients with the forced frequency (depth of fluid $h=1.0 \mathrm{~cm}$, radius of the vessel $R=7.5 \mathrm{~cm}$, forced amplitude $A=11.4 \mu \mathrm{m}$, the surface tension of the fluid $\Gamma=0.072716 \mathrm{~N} / \mathrm{m}$, the density of fluid $\rho=10^{3} \mathrm{Kg} / \mathrm{m}^{3}$ ). (a) Variation of the coefficient $M_{1}$ with forced frequency, (b) variation of the coefficient $M_{2}$ with forced frequency.

imaginary parts of $p(\tau)$, divide (2.14) into real and imaginary parts, it yields the following nonlinear ordinary differential equation groups

$$
\begin{aligned}
& \frac{\mathrm{d} p_{1}(\tau)}{\mathrm{d} \tau}=M_{1} p_{2}(\tau)\left[p_{1}^{2}(\tau)+p_{2}^{2}(\tau)\right]+M_{2}\left[p_{1}(\tau) \sin (2 \sigma \tau)-p_{2}(\tau) \cos (2 \sigma \tau)\right], \\
& \frac{\mathrm{d} p_{2}(\tau)}{\mathrm{d} \tau}=-M_{1} p_{1}(\tau)\left[p_{1}^{2}(\tau)+p_{2}^{2}(\tau)\right]-M_{2}\left[p_{1}(\tau) \cos (2 \sigma \tau)+p_{2}(\tau) \sin (2 \sigma \tau)\right] .
\end{aligned}
$$

For prescribed initial condition, the solutions $p_{1}(\tau)$ and $p_{2}(\tau)$ of $(2.15),(2.16)$ can be obtained via numerical computation, and then two-order surface wave displacement $(2.8)$ can be obtained.

\subsection{The comparison of the coefficients of the amplitude equation}

In this subsection, we gave the computational results describing the variation of the coefficients $M_{1}$ and $M_{2}$ of (2.14) with the forced frequency in Fig. 2 under the circumstances of having surface tension and having no surface tension respectively.

It can be seen from Fig. 2(a) that the nonlinear coefficient $M_{1}$ changed largely under the condition of having surface tension than those of having no surface tension. From the point of view of the equilibrium, the solution of amplitude equation (2.14) with the surface tension is smaller than that of having no surface tension. These means the surface tension has the property, which causes the free surface returning to equilibrium location.

Fig. 2(b) demonstrated that the external forced coefficient $M_{2}$ increases with the forced frequency incorporating both surface tension and no surface tension (we only consider the absolute value of $M_{2}$ and the coefficient $M_{2}$ was multiplied by $\varepsilon^{2}$ ). However, the $M_{2}$ changes more slowly when the surface tension is taken into account than that of having no surface tension. It still proved the above character of the surface tension.

In fact, the damping will appear in actual physical system due to dissipation of viscosity of the fluid, and it has some effects on the mode selection. In the next section, we will study the vertical forced surface waves in a weakly viscid fluid, and determine the analytical expression of damping coefficient. Then the dispersive relation (2.11) and nonlinear amplitude equation (2.14) are modified by adding damping. Finally, we study the stability of this modified equation.

\section{The theory of vertical forced surface waves in weakly viscid fluid}

\subsection{Governing equations and boundary conditions}

Now we consider the surface wave excited by vertical oscillation of a circular cylindrical basin filled with weakly viscous fluids. All parameters and the choice of the coordinate system are the same as Fig. 1. We suppose the fluid is incompressible, so the velocity of the fluid satisfies the condition of zero divergence

$$
\nabla \cdot \vec{v}=0
$$


here $\vec{v}=\vec{v}(r, \theta, z, t)$ is the velocity field of fluid and obeying the Navier-Stokes equation

$$
\frac{\partial \vec{v}}{\partial t}+\vec{v} \cdot \nabla \vec{v}=-\frac{1}{\rho} \nabla p-g(t) \vec{e}_{z}+v \nabla^{2} \vec{v},
$$

where $\rho$ is the mass density, $p$ is the pressure, $v$ is the kinematic viscosity and $g(t)$ is the effective gravity, i.e. $g$ minus the acceleration of the vibrating tank

$$
g(t)=g-\ddot{z}_{0}=g+4 A \omega_{0}^{2} \cos 2 \omega_{0} t .
$$

In order to allow for a unique solution of the Navier-Stokes equation, one must specify boundary conditions. The appropriate boundary condition at the free surface $z=\eta(r, \theta, t)$ is the kinematic condition

$$
\left.w(r, \theta, z, t)\right|_{z=\eta}=\frac{\partial \eta}{\partial t}+\vec{v} \cdot \nabla \eta,
$$

where $\left.\right|_{\eta}$ means the evaluation at $z=\eta$, and $w$ denotes the velocity in the $z$ direction. At the liquid-air interface $z=\eta(r, \theta, t)$, the net force per unit area is balanced by the surface tension $\Gamma$, thus we have

$$
\left(T_{j k}^{\mathrm{air}}-T_{j k}\right) \cdot n_{k}=\Gamma\left(\frac{1}{R_{1}}+\frac{1}{R_{2}}\right) n_{j},
$$

here $T_{j k}^{\text {air }}$ and $T_{j k}$ denote the stress tensor of the air and liquid respectively, $\vec{n}$ is the unit outward normal to the surface, $\Gamma$ the surface tension, $R_{1}$ and $R_{2}$ the radius of curvature of the surface calculated using the principal axes. Because the dynamic viscosity of air is smaller than that of a liquid, the stress tensor of the air above the liquid layer can be approximated by a constant atmospheric pressure. Thus we have

$$
\left\{\begin{array}{l}
T_{j k}^{\text {air }} \approx-p_{0} \delta_{j k}, \\
T_{j k}=-p \delta_{j k}+\rho v\left(\frac{\partial v_{k}}{\partial x_{j}}+\frac{\partial v_{j}}{\partial x_{k}}\right),
\end{array}\right.
$$

here the $p_{0}$ is atmospheric pressure which we assume constant and $\delta_{j k}$ is Kronecker Delta operator. At the bottom and on the side-wall of the container, one must use the no-slip condition

$$
\vec{v}(r, \theta, z, t)=0 .
$$

For sufficiently small drive amplitude $A$, the fluid remains at rest and the surface is flat. It is therefore convenient to introduce the pressure function $\Pi(r, \theta, z, t)$ from (3.5) and (3.6), and let

$$
\Pi(r, \theta, z, t)=p(r, \theta, z, t)-p_{0}+\rho g(t) z
$$

the Navier-Stokes equation (3.2) is changed to

$$
\frac{\partial \vec{v}}{\partial t}+\vec{v} \cdot \nabla \vec{v}=-\frac{1}{\rho} \nabla \Pi+\nu \nabla^{2} \vec{v} .
$$

At the free surface, (3.5) can be divided into the normal part, which is perpendicular to the free surface

$$
\left.\Pi(r, \theta, z, t)\right|_{z=\eta}-\rho g(t) \eta-2 \rho v \frac{\partial w}{\partial z}=\Gamma\left(\frac{1}{R_{1}}+\frac{1}{R_{2}}\right),
$$

and the tangential part of the free surface

$$
\rho v\left(\frac{\partial u}{\partial z}+\frac{\partial w}{\partial r}\right)=\rho v\left(\frac{1}{r} \frac{\partial w}{\partial \theta}+\frac{\partial v}{\partial z}\right)=0,
$$

where $u$ and $v$ denote the velocity components in $r$ and $\theta$ direction respectively. In the case of ideal fluids, if we retain only the linear terms of (3.9), and suppose the wave motion is irrotational, we have

$$
\Pi(r, \theta, z, t)=-\rho \frac{\partial \phi}{\partial t},
$$

where $\phi(r, \theta, z, t)$ is inviscid velocity potential. Now we take Eq. (3.12) as the approximate function when we consider weakly viscous liquid as followings.

As for the linear viscous problem, it is reasonable to separate the velocity into two components

$$
\vec{v}=\nabla \phi+\vec{U},
$$


namely, potential flow and boundary layer velocity. Here the velocity vector $\vec{U}$ is relevant to boundary layers while the potential part is important in the rest of the fluid. Substituting (3.12), (3.13) into (3.9), and ignoring the nonlinear terms, we obtain following diffusive equation with respect to the velocity of boundary layer

$$
\frac{\partial \vec{U}}{\partial t}=v \nabla^{2} \vec{U}
$$

Take Eq. (3.13) into (3.1), boundary layer velocity satisfies zero divergence condition

$$
\nabla \cdot \vec{U}=0
$$

Substituting (3.13) into (3.1), (3.4), (3.10), (3.11) and (3.7), neglecting all nonlinear terms, incompressible condition (3.1) can be expressed as

$$
\frac{\partial^{2} \phi}{\partial r^{2}}+\frac{1}{r} \frac{\partial \phi}{\partial r}+\frac{1}{r^{2}} \frac{\partial^{2} \phi}{\partial \theta^{2}}+\frac{\partial^{2} \phi}{\partial z^{2}}=0, \quad-h \leqslant z \leqslant \eta(r, \theta, t), 0<r<R .
$$

The boundary conditions at the free surface

$$
\begin{aligned}
& \frac{\partial \eta}{\partial t}-\frac{\partial \phi}{\partial z}=W, \quad z=\eta(r, \theta, t) \\
& \frac{\partial \phi}{\partial t}+g\left(1+\frac{4 A \omega_{0}^{2}}{g} \cos 2 \omega_{0} t\right) \eta+2 v\left(\frac{\partial^{2} \phi}{\partial z^{2}}+\frac{\partial W}{\partial z}\right)=0, \quad z=\eta(r, \theta, t), \\
& \rho \nu\left(\frac{\partial u}{\partial z}+\frac{\partial w}{\partial r}\right)=\rho v\left(\frac{1}{r} \frac{\partial w}{\partial \theta}+\frac{\partial v}{\partial z}\right)=0, \quad z=\eta(r, \theta, t)
\end{aligned}
$$

and no-slip condition on the side-wall and at the bottom

$$
\nabla \phi+\vec{U}=0, \quad z=-h \text { and } r=R
$$

must be satisfied. Here $W$ is the component of boundary layer velocity $\vec{U}$ in $z$ direction. Eqs. (3.14)-(3.20) are elementary linearized equations in the viscous fluids. Our aim is to study weakly viscous surface waves via solving above potential flow and boundary layer equations.

\subsection{Nondimensional equations and establishment of curve coordinates}

Taking the radius $R$ of the tank as the length scale, nondimensionalizing all related independent variables and unknown variables, the following scalings are adopted,

$$
\begin{aligned}
& z^{*}=z / R, \quad r^{*}=r / R, \quad \eta^{*}=\eta / R, \quad t^{*}=t / \sqrt{R / g}, \quad \phi^{*}=\phi /(R \sqrt{g R}), \quad A^{*}=A / R, \\
& \omega_{0}^{*}=\omega_{0} / \sqrt{g / R}, \quad \varepsilon^{*}=4 A \omega_{0}^{2} / g, \quad \nu^{*}=v /\left(\varepsilon^{* 2} R \sqrt{g R}\right), \quad \vec{U}^{*}=\vec{U} / \sqrt{g R} .
\end{aligned}
$$

Similarly, the asterisks denote dimensionless quantities and that the asterisks are subsequently dropped. We have supposed that the viscosity is weak, and has been scaled as $\varepsilon^{2}$ in Eq. (3.21) in order to facilitate the theoretical analysis in mathematics. Substituting (3.21) into (3.14)-(3.20), we obtain following dimensionless governing equations

$$
\frac{\partial^{2} \phi}{\partial r^{2}}+\frac{1}{r} \frac{\partial \phi}{\partial r}+\frac{1}{r^{2}} \frac{\partial^{2} \phi}{\partial \theta^{2}}+\frac{\partial^{2} \phi}{\partial z^{2}}=0, \quad-h / R \leqslant z \leqslant \eta(r, \theta, t), 0<r<1,
$$

boundary conditions at free surface $z=\eta(r, \theta, t)$

$$
\begin{aligned}
& \frac{\partial \eta}{\partial t}-\frac{\partial \phi}{\partial z}=W, \quad z=\eta(r, \theta, t) \\
& \left\{\begin{array}{l}
\varepsilon^{2}\left(2 \frac{\partial^{2} \phi}{\partial r \partial z}+\frac{\partial W}{\partial r}\right)+\varepsilon^{2} \frac{\partial U}{\partial z}=0, \\
\varepsilon^{2}\left(\frac{2}{r} \frac{\partial^{2} \phi}{\partial \theta \partial z}+\frac{1}{r} \frac{\partial W}{\partial \theta}\right)+\varepsilon^{2} \frac{\partial V}{\partial z}=0, \\
\left.\frac{\partial \phi}{\partial t}+\left(1+\varepsilon \cos 2 \omega_{0} t\right) \eta+2 \nu \varepsilon^{2}\left(\frac{\partial^{2} \phi}{\partial z^{2}}+\frac{\partial W}{\partial z}\right)=0, \quad z=\eta(r, \theta, t), t\right)
\end{array}\right.
\end{aligned}
$$




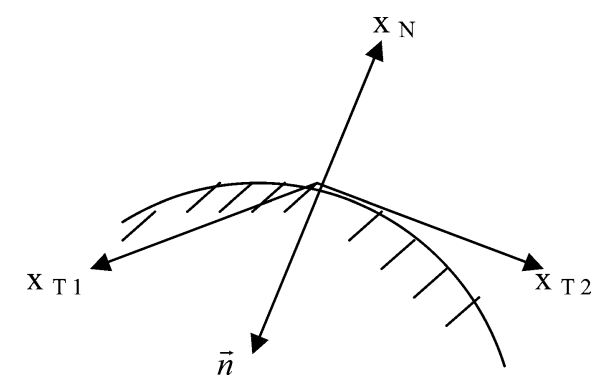

Fig. 3. Curve coordinates of the boundary layer.

and boundary conditions on the side-wall and at the bottom of the vessel

$$
\nabla \phi+\vec{U}=0, \quad z=-h / R \text { and } r=1 .
$$

The boundary layer equations are

$$
\begin{aligned}
& \frac{\partial \vec{U}}{\partial t}=\varepsilon^{2} v \nabla^{2} \vec{U}, \\
& \nabla \cdot \vec{U}=0,
\end{aligned}
$$

where $U$ and $V$ are velocity components of the boundary layer in $r$ and $\theta$ directions respectively.

Next we establish a boundary layer coordinate system. Recalling that the order of viscosity $v$ is $\mathrm{O}\left(\varepsilon^{2}\right)$, and the thickness $\delta$ of an oscillatory boundary layer has the order $\mathrm{O}\left\{(2 v / \Omega)^{1 / 2}\right\}$, so the order of the boundary layer thickness is $\mathrm{O}(\varepsilon)$. A boundary layer coordinate is introduced as shown in Fig. 3. Where $x_{N}$ is the normal direction pointing into the fluid from the wall, hence, opposite to $\vec{n} . x_{T 1}$ and $x_{T 2}$ determined tangential plane that is perpendicular to the normal direction $x_{N}$. So $x_{N}, x_{T 1}$ and $x_{T 2}$ form a locally rectangular coordinate system.

We introduce a new boundary layer variable

$$
\varsigma=\frac{x_{N}}{\varepsilon},
$$

then the continuity equation (3.28) in terms of the local coordinate is

$$
-\frac{\partial \vec{n} \cdot \vec{U}}{\partial \varsigma}+\varepsilon\left(\frac{\partial U_{T_{1}}}{\partial x_{T_{1}}}+\frac{\partial U_{T_{2}}}{\partial x_{T_{2}}}\right)=0
$$

and boundary layer equation (3.27) becomes

$$
\frac{\partial \vec{U}}{\partial t}=v \frac{\partial^{2} \vec{U}}{\partial \varsigma^{2}},
$$

due to the supposition of weak viscosity.

In the next section, we will solve potential equations (3.22) with boundary condition (3.23)-(3.26) and boundary layer equations (3.30), (3.31) by two-time scale perturbation expansions.

\subsection{The solution of the problem and determination of the damping coefficient}

We expand the related variables into power series of the small parameter $\varepsilon$. A slowly varying time scale $\tau$ is introduced, such that

$$
\tau=t \varepsilon \text {. }
$$

Expanding velocity potential $\phi$, free surface displacement $\eta$ and velocity vector $\vec{U}$ of the boundary layer into

$$
\left\{\begin{array}{l}
\phi=\phi_{0}(r, \theta, z, t, \tau)+\varepsilon \phi_{1}(r, \theta, z, t, \tau)+\cdots, \\
\eta=\eta_{0}(r, \theta, t, \tau)+\varepsilon \eta_{1}(r, \theta, t, \tau)+\cdots, \\
\vec{U}=\vec{U}_{0}(r, \theta, z, t, \tau)+\varepsilon \vec{U}_{1}(r, \theta, z, t, \tau)+\cdots,
\end{array}\right.
$$

and then expanding (3.23) and (3.25) into Taylor series about $z=0$. Provided that we neglect higher order terms $\mathrm{O}\left(\varepsilon^{2}\right)$, substitute (3.32), (3.33) into (3.22)-(3.26), compare with the coefficients the small parameter $\varepsilon^{i}$ at the two hands of all equations, 
and obtain each order governing equations and boundary conditions for both potential flow and boundary layer. The boundary layer is composed of side-wall, bottom and meniscus (this region is defined as the overlap between free surface and the side wall boundary layer).

We can obtain external potential solution and internal boundary layer solution of $\mathrm{O}\left(\varepsilon^{0}\right)$ problem by separation of variables. Similar to the method in inviscid fluid, the solvability condition of $\mathrm{O}\left(\varepsilon^{1}\right)$ approximation can be given. The detailed process is ignored in this paper, and only the important results are giving in followings. The derived amplitude equation associated with slow varying time is

$$
\frac{\mathrm{d} p(\tau)}{\mathrm{d} \tau}=-\mathrm{i} \alpha \bar{p}(\tau)+\beta p(\tau)
$$

here $\alpha$ is positive real number, represents the influence of vertically external excitation, and $\beta$ denotes the complex damping coefficient. The detailed expressions of $\alpha$ and $\beta$ are

$$
\alpha=\frac{\Omega}{4}, \quad \beta=\beta_{W}+\beta_{B}+\beta_{M},
$$

where $\beta_{W}, \beta_{B}$, and $\beta_{M}$ represent the viscous damping in the side-wall, the bottom boundary layer, and the meniscus region respectively, and they can be written as

$$
\begin{aligned}
& \beta_{W}=\frac{\lambda[\sinh (2 \lambda h / R)+2 \lambda h / R]}{8 \Omega \cosh ^{2}(\lambda h / R)} \sqrt{\frac{2 v}{\Omega}}(1+\mathrm{i}), \\
& \beta_{B}=\frac{\lambda^{2}}{4 \Omega \cosh ^{2}(\lambda h / R)} \sqrt{\frac{2 v}{\Omega}}(1+\mathrm{i}), \\
& \beta_{M}=\frac{\lambda^{2} \Omega}{2\left(\lambda^{2}-m^{2}\right)} \sqrt{\frac{2 v}{\Omega}}(1+\mathrm{i}) .
\end{aligned}
$$

The formulations for the damping rates (3.36)-(3.38) are different from those found by Case and Parkinson [27], where they calculated the damping rates by energy dissipation method. The expressions for damping rates in [27] contain the damping in the body of the liquid, but our result does not include this term. Case and Parkinson [27] noted that the damping in the body of the liquid is small compared to the side-wall damping, particularly for large cylinders. In addition, our current results include the damping in the meniscus region, which is overlooked in [27]. As discussed by Mei and Liu [25], the side-wall layer receives power from waves through the meniscus boundary layer. In summary, neither the boundary layer method of the present analysis nor the energy methods of previous analyzes sufficiently accounts for the discrepancies between theory and experiment noted by Keulegan [31]. A comparison of damping rates in present analysis with those in [27] can be found in followings.

In order to make clear the physical meaning of the damping coefficient, Fig. 4 illustrates the dimensional damping coefficient as a function of viscosity. It can be shown from Fig. 4 that the damping coefficients of the side-wall, the bottom and the meniscus regions increase with the viscosity. This obvious result can be easily seen from Eqs. (3.36)-(3.38).

Fig. 5 shows the variations of damping coefficient with different depth of the liquid layer. With the increase in the depth of the liquid, the area of the side-wall boundary layer increases accordingly, so the damping on the side-wall will increase too.

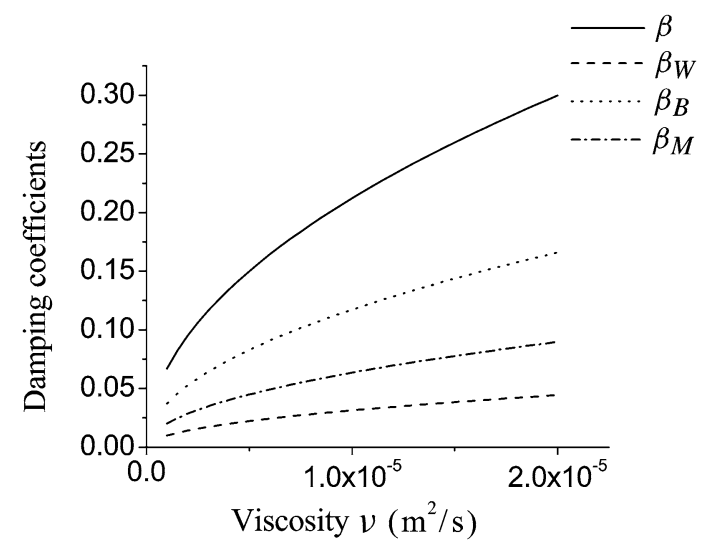

Fig. 4. Variation of damping with viscosity (forced amplitude $A=11.4 \mu \mathrm{m}$, forced frequency $f=8.5 \mathrm{~Hz}$, radius of the vessel $R=7.5 \mathrm{~cm}$, depth of the fluid $h=1.0 \mathrm{~cm}$ ).

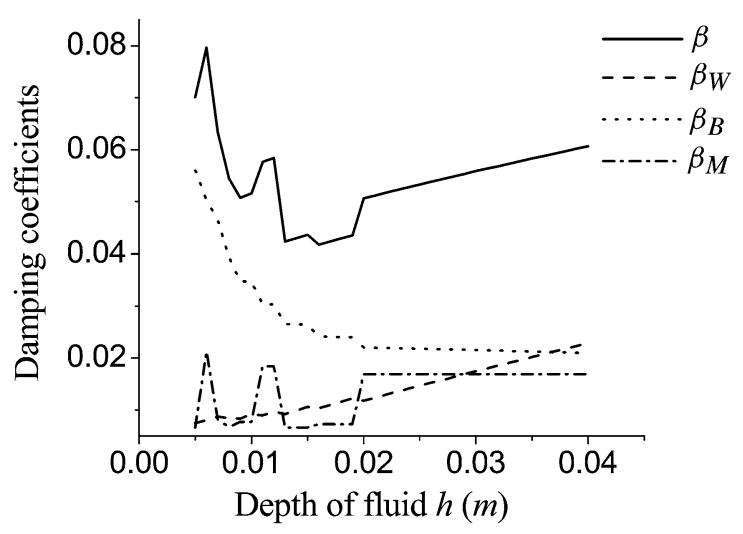

Fig. 5. Variation of damping with the depth of fluid (forced amplitude $A=11.4 \mu \mathrm{m}$, forced frequency $f=9 \mathrm{~Hz}$, radius of the vessel $R=7.5 \mathrm{~cm}$, viscosity coefficient $v=10^{-6} \mathrm{~m}^{2} / \mathrm{s}$ ). 


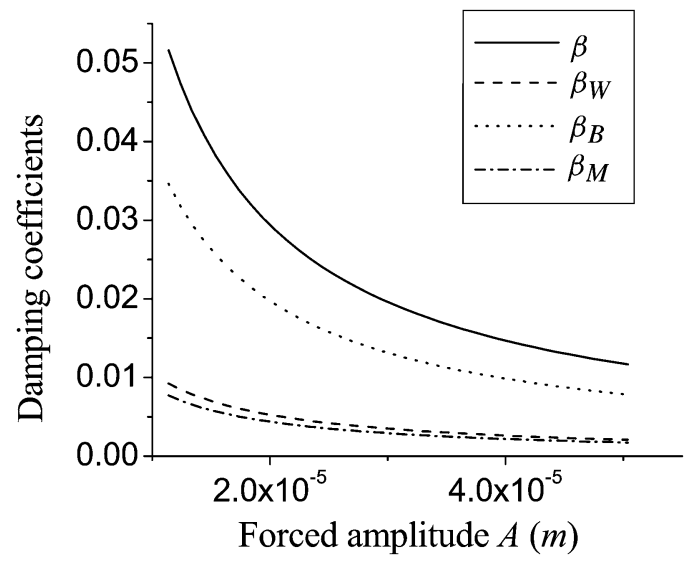

Fig. 6. Variation of damping with forced amplitude (forced frequency $f=9 \mathrm{~Hz}$, radius of the vessel $R=7.5 \mathrm{~cm}$, depth of fluid $h=1.0 \mathrm{~cm}$, viscosity coefficient $v=10^{-6} \mathrm{~m}^{2} / \mathrm{s}$ ).

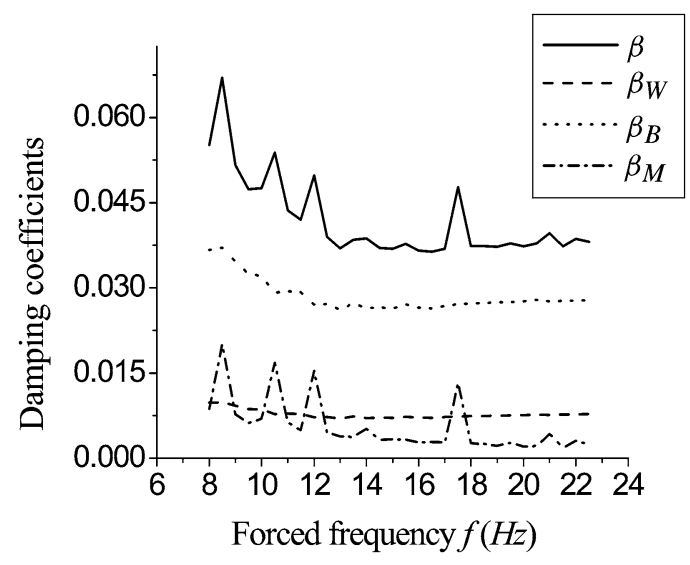

Fig. 7. Variation of damping with forced frequency (forced amplitude $A=11.4 \mu \mathrm{m}$, radius of the vessel $R=7.5 \mathrm{~cm}$, depth of fluid $h=1.0 \mathrm{~cm}$, viscosity coefficient $\left.v=10^{-6} \mathrm{~m}^{2} / \mathrm{s}\right)$.

However, the damping of the bottom decreases with the increase in the depth of the fluid. As the fluid layer becomes shallow, the whole damping is primarily concentrated on the bottom boundary layer. In the region of meniscus, the damping exhibits a jump in a certain depth. This is mainly due to the variability of mode selection, which can change the value of denominator in (3.38). When the depth of the fluid exceeds $2.0 \mathrm{~cm}$, the damping in meniscus increases with the depth of the fluid.

The dependence of the damping coefficient upon the forced amplitude is shown in Fig. 6. Clearly, the damping decreases with the increase of the forced amplitude. The reason is that the energy entering into the system by external excitation is more than that of dissipation caused by viscosity. Under this condition, the energy will be accumulated and the surface wave becomes unstable.

Fig. 7 illustrates the variation of damping coefficient with forced frequency. As indicated in the figure, the damping decreases gradually with the increase of forced frequency. The reasons are similar to that for Fig. 6 . The damping of the meniscus is influenced by the selected mode of the surface wave as in Fig. 5, while some jump phenomena appear.

\subsection{Comparison with Case and Parkinson (1957)}

Case and Parkinson [27] studied the damping of surface waves for incompressible fluid in a fixed cylindrical container theoretically and experimentally. The theoretical and experimental results were found to be in good agreement. We will compare our present theory with that of Case and Parkinson though the mechanisms of producing surface waves are different each other. The comparison of the damping in Case and Parkinson's results with that of our present theoretical results in Fig. 8.

It can be seen from Fig. 8 that our theoretical results are qualitatively accorded with those of Case and Parkinson though our theoretical damping is large. We believe that the discrepancy between them is reasonable. First reason is that our theoretical results contain the damping in meniscus region, which is important to total damping distribution from Figs. 4-7. However, Ref. [27] overlooked the meniscus damping in their analysis. Second reason is likely that dimensional viscosity coefficient $v$ in Eq. (3.21) is divided by small dimensionless parameter $\varepsilon^{2}$ when we scale the viscosity. Thus dimensionless damping rates in Eqs. (3.36)-(3.38) will be increased due to the larger viscosity $v$. The parameter $\varepsilon$ is related to forced parameters, e.g. excited amplitude and frequency, so the damping of the surface waves is related to excited amplitude and frequency. Moreover, the surface waves would disappear immediately if the external excitation stops in our experimental observation. This suggests the damping is larger in the vertically forced surface waves than that of Ref. [27].

\subsection{The effect of surface tension and weak viscosity on mode selection}

Henderson [32] pointed out that the real and the imaginary part of the (3.35) mean the value of the damping and frequency shift respectively. The damping causes the attenuation of the surface wave, while the frequency shift changes the natural frequency of the surface wave. The natural frequency (2.11) of surface wave, which includes the effect of surface tension, can be modified by introducing the imaginary part $\beta_{2}$ of damping coefficient $\beta$, namely

$$
\widehat{\Omega}=\Omega-\beta_{2},
$$




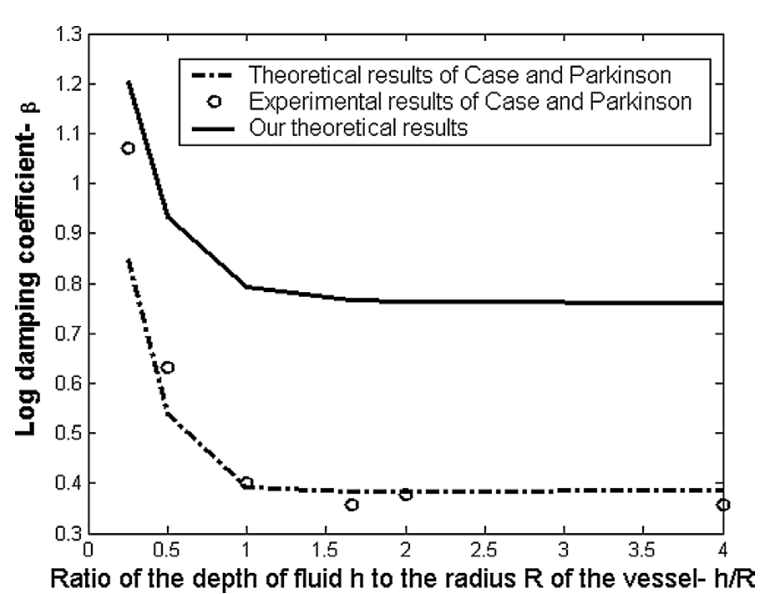

(a)

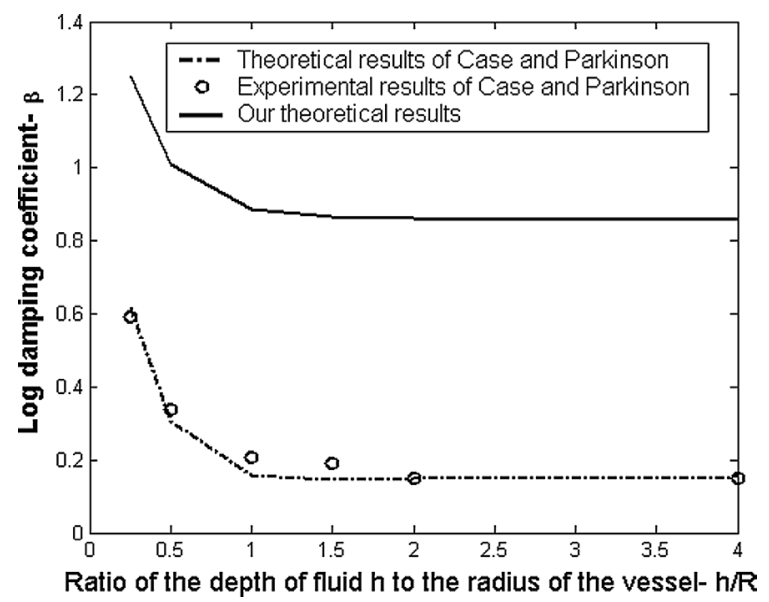

(b)

Fig. 8. Comparison of the damping in Case and Parkinson's results with that of our present theoretical results (the forcing amplitude $A=11.4 \mu \mathrm{m}$, the viscosity coefficient $v=10^{-6} \mathrm{~m}^{2} / \mathrm{s}$, the surface tension of the fluid $\Gamma=0.072716 \mathrm{~N} / \mathrm{m}$, the density of fluid $\rho=10^{3} \mathrm{Kg} / \mathrm{m}^{3}$ ). (a) $R=1.5$ in. (b) $R=3.0$ in.

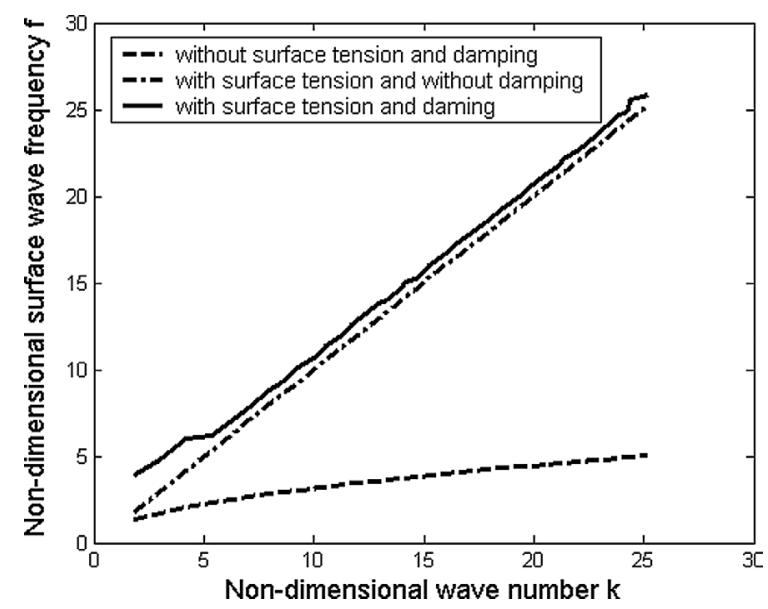

Fig. 9. Variation of dimensionless natural frequency and modified frequency with dimensionless wave number (depth of fluid $h=10 \mathrm{~cm}$, radius of the vessel $R=7.5 \mathrm{~cm}$, viscosity coefficient $\nu=10^{-6} \mathrm{~m}^{2} / \mathrm{s}$, surface tension of the fluid $\Gamma=0.072716 \mathrm{~N} / \mathrm{m}$, the density of fluid $\left.\rho=10^{3} \mathrm{Kg} / \mathrm{m}^{3}\right)$.

where

$$
\begin{aligned}
& \Omega=\sqrt{\lambda \tanh (\lambda h / R)\left(1+\frac{\Gamma}{\rho} \lambda^{2}\right)}, \\
& \beta_{2}=\left[\frac{\lambda[\sinh (2 \lambda h / R)+2 \lambda h / R]}{8 \Omega \cosh ^{2}(\lambda h / R)}+\frac{\lambda^{2}}{4 \Omega \cosh ^{2}(\lambda h / R)}+\frac{\lambda^{2} \Omega}{2\left(\lambda^{2}-m^{2}\right)}\right] \sqrt{\frac{2 \nu}{\Omega}} .
\end{aligned}
$$

In order to show the influence of surface tension and damping on the pattern selection, we present a dimensionless surface wave frequency versus dimensionless wave number curve in Fig. 9. It can be seen from Fig. 9 that when the forcing frequency is low, the viscosity of the fluid is a prominent factor for the mode selection. However, when the forcing frequency is high, the surface tension of the fluid is the prominent. This result shows that modified frequency much reasonably closes to experimental results than that of Refs. [22,23]. 


\subsection{The characters of the surface waves}

\subsubsection{The relation between surface wave mode and forced frequency}

If the forced amplitude is prescribed, different forced frequencies will produce different surface wave modes. We know the small parameter $\varepsilon$ is associated with forced frequency and amplitude, so when the forced frequency increases gradually, the forced amplitude must be small enough to ensure the parameter $\varepsilon$ is small. When forced amplitude is 11.4 micron and forced frequencies are 9.65, 11.99, 12.56 and $19.72 \mathrm{~Hz}$, the contours of the free surface displacements and corresponding threedimensional surface figures determined by (2.8), are plotted in Fig. 10 at prescribed time $t=215.9 \mathrm{~s}$ with the effect of surface tension and weak viscosity considered. In Fig. 10 and following related figures, the solid or dashed lines of the contours denote the position of free surface is above or blow the equilibrium surface at this time. The parametrical couple of $(m, n)$ means that there are $m$ wave-crests in circumferential direction and $n$ zero points in radical direction. We can see from Fig. 10 that the surface wave modes become more and more complex with the increase of the forced frequency.

\subsubsection{Standing surface wave property}

We will validate theoretically that the excited surface waves have the character of standing waves. The numbers of nodes and their spatial distributions of the surface waves are very important to understand the motion of surface waves. The comparison between the contours of surface wave displacement of $(9,6)$ mode in experiment [19-21] and theoretical result from $(2.8)$ at $t=244.29 \mathrm{~s}$ is made in Fig. 11. Moreover, spatial distribution and time evolution of this mode are presented. It can be seen from Fig. 11 that these two figures are much similar. There are 9 wave peaks in circumferential direction and 6 zero points in radical direction. The forced frequency in experiment is $52 \mathrm{~Hz}$, and theoretical forced frequency is $29.01 \mathrm{~Hz}$ when the surface tension and weak viscosity are considered. The difference is still very large. However, the forced frequency in this paper is much closer to experimental result than that of $19.7 \mathrm{~Hz}$ in [23] in inviscid fluid. The discrepancy in theory and experiment maybe caused by moving contact line between side-wall and surface of fluid, wetting phenomenon, and mode competition, etc.

Fig. 12 illustrates the evolution of the surface wave displacement (2.8) with nondimensional variable $r(\theta=4 \pi / 3)$ and spatial coordinate $\theta(r=0.5)$ at different time. It can be seen from Fig. 12 that the positions of nodes are unchanged, only the amplitude of the surface wave changes up and down, which explains the property of the standing surface wave.

The three-dimensional surface figures of the free surface displacement at different time are illustrated in Fig. 13. When $t=0.563$ (s) in Fig. 13(a), the displacement of surface wave in the point of $S$ obtains its positive maximum, where the line $S$ denotes the surface wave in this specific position at this time. With the development of time, as showed in Fig. 13(b), the displacement of location $S$ decreases. In next time $t=0.872$ (s), as showed in Fig. 13(c), the displacement of the surface wave in $S$ location changes from above equilibrium to below it and the displacement becomes negative. With the evolution of time, as showed in Fig. 13(d), the displacement in $S$ location becomes the negative maximum. With the development of time, the displacement of $S$ will decrease and then obtain its positive maximum. The trait of standing surface wave is proved theoretically.

It is necessary to point out that the location $S$ is only a typical surface wave location, and other displaces still have the same character as the line $S$. Moreover, $(9,6)$ mode is a special case for surface wave, and other modes of the surface waves can be studied by the same approach.

\section{Instability theory of vertical forced surface waves}

\subsection{Modified amplitude equation}

From the point of view of physics, the conjugate term of the nonlinear amplitude equation (2.14) means the energy entered via external excitation. If we do not consider the viscous dissipation, the amplitude of the surface wave would increase gradually. In order to keep forced energy in equilibrium with that of the viscous dissipation, we will modify this equation by intruding the damping term and discuss its instability.

Substituting the real part $\beta_{1}$ of the damping coefficient $\beta$ into the left hand of nonlinear evolution equation (2.14), we obtain following modified equation

$$
\mathrm{i}\left(\frac{\mathrm{d}}{\mathrm{d} \tau}+\beta_{1}\right) p(\tau)=M_{1} p^{2}(\tau) \bar{p}(\tau)+M_{2} \mathrm{e}^{2 \mathrm{i} \sigma \tau} \bar{p}(\tau),
$$

where $\sigma, \beta_{1}, M_{1}$ and $M_{2}$ are real number, $\beta_{1}=\operatorname{Re}(\beta)>0$. From (3.35)-(3.38), we know $\beta_{1}=\beta_{2}$ and its expression can be given by (3.40). For the convenience of solving the modified amplitude equation (4.1), we made a transformation for a unknown function $p(\tau)$, let

$$
q(\tau)=p(\tau) \mathrm{e}^{-\mathrm{i} \sigma \tau},
$$



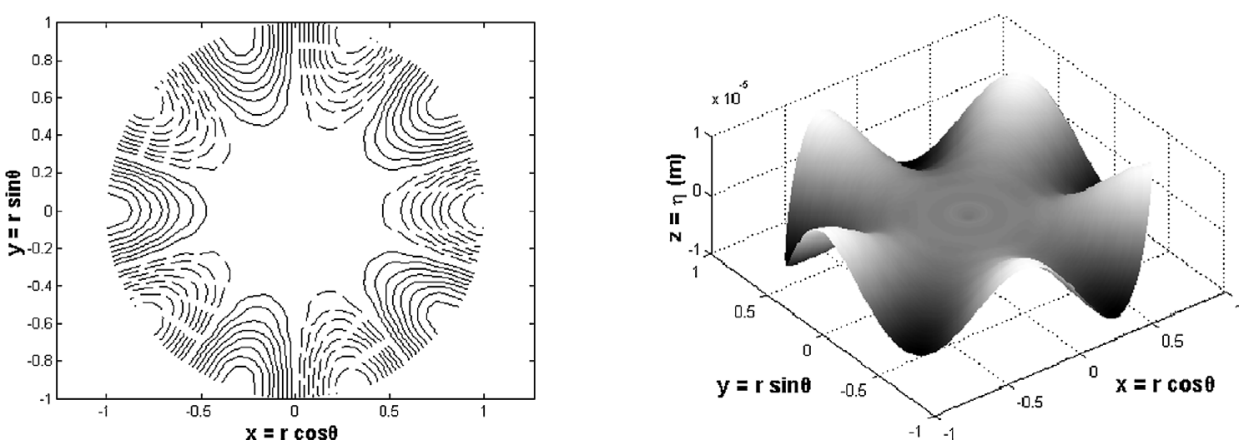

(a)
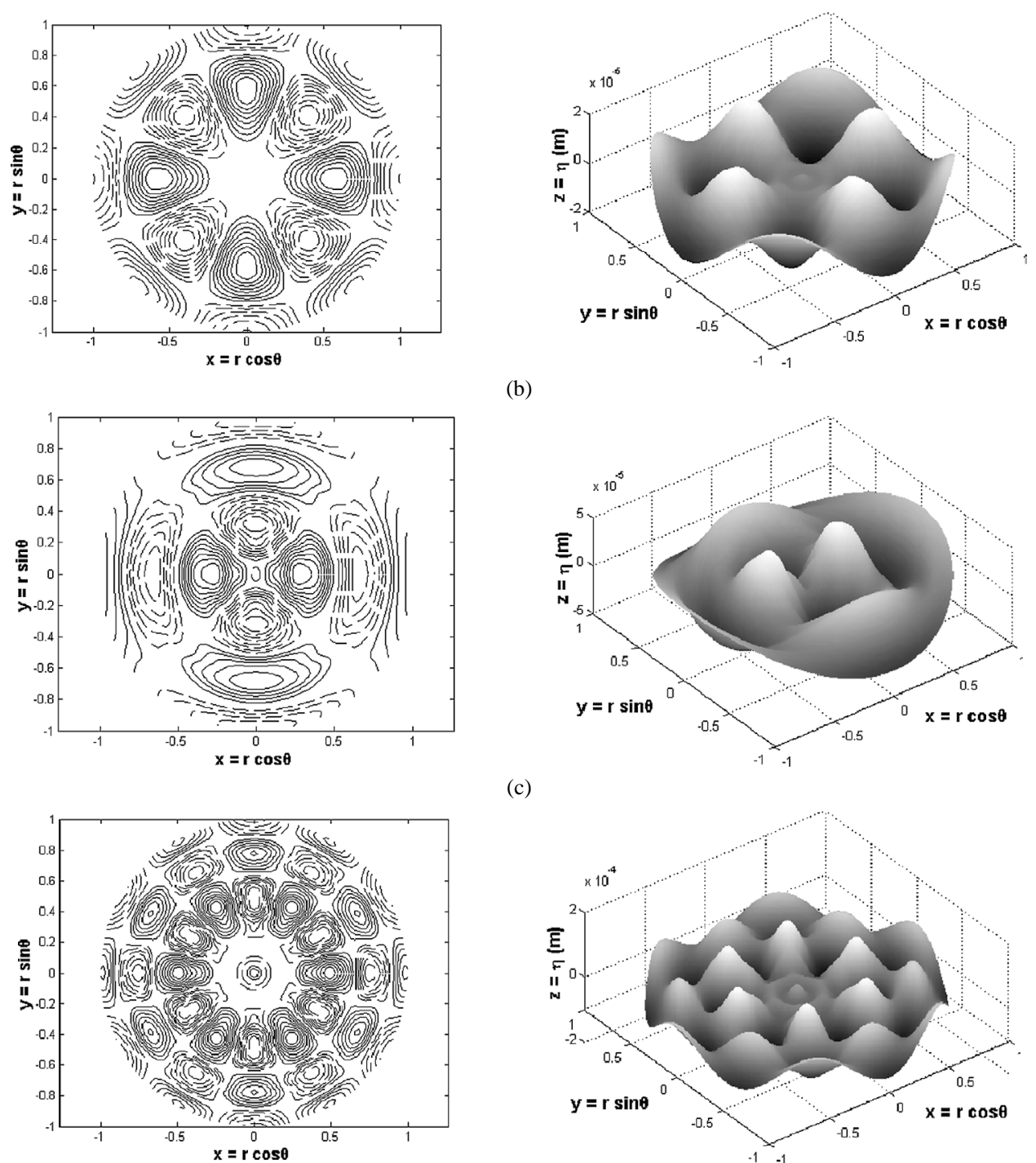

(c)

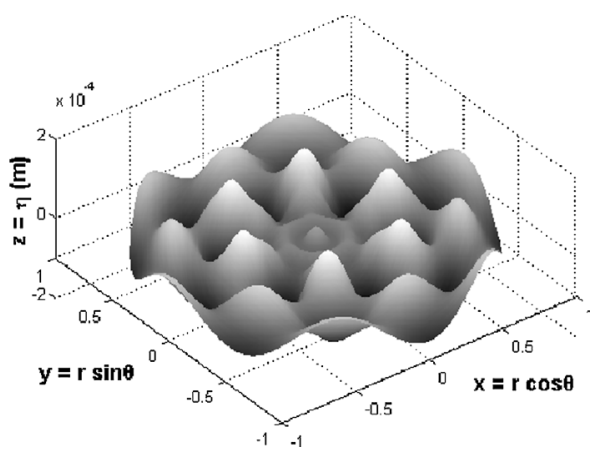

(d)

Fig. 10. Contours (left) and three-dimensional modes (right) of the surface wave at different forced frequencies (depth of fluid $h=1.0 \mathrm{~cm}$, radius of vessel is $R=7.5 \mathrm{~cm}$, forcing amplitude $A=11.4 \mu \mathrm{m}$, viscosity coefficient $\nu=10^{-6} \mathrm{~m}^{2} / \mathrm{s}$, surface tension of the fluid $\Gamma=0.072716 \mathrm{~N} / \mathrm{m}$, the density of fluid $\rho=10^{3} \mathrm{Kg} / \mathrm{m}^{3}$ ). (a) Forced frequency $f=9.65 \mathrm{~Hz}$, the mode of surface wave is $(5,1)$, (b) forced frequency $f=11.99 \mathrm{~Hz}$, the mode of surface wave is $(4,2)$, (c) forced frequency $f=12.56 \mathrm{~Hz}$, the mode of surface wave is $(2,3)$, (d) forced frequency $f=19.72 \mathrm{~Hz}$, the mode of surface wave is $(6,3)$. 


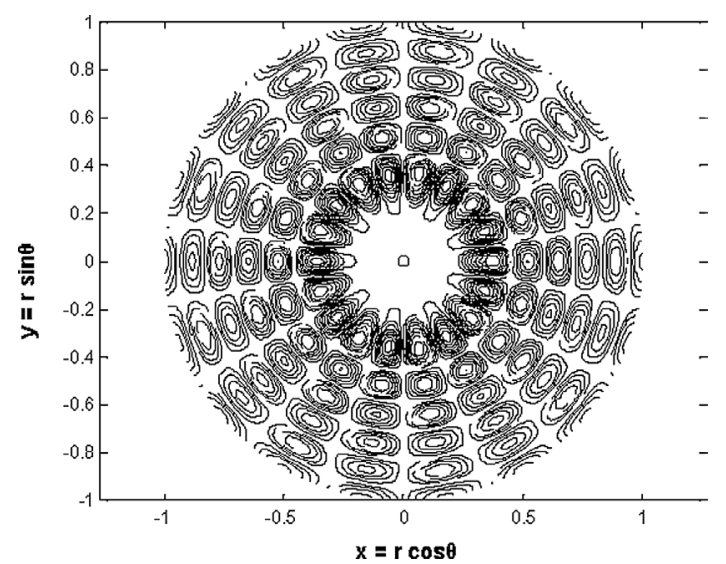

(a)

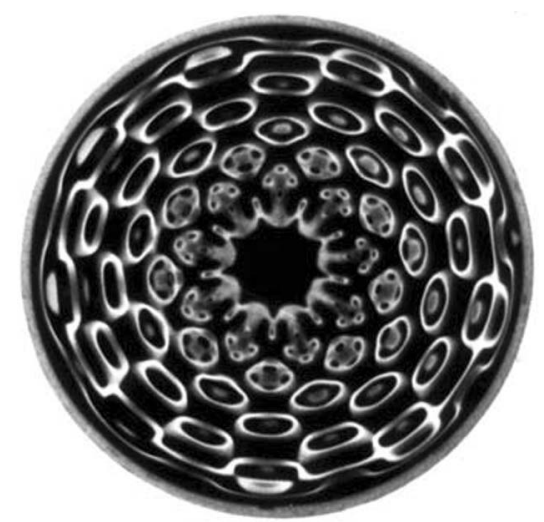

(b)

Fig. 11. Contours of the $(9,6)$ mode in both theory and experiment. (a) Theoretical contour of $(9,6)$ mode, $(b)$ experimental contour of $(9,6)$ mode.

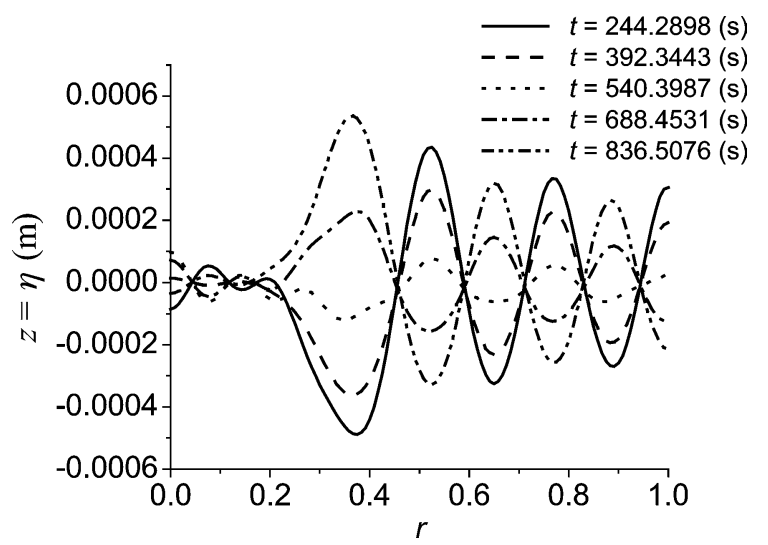

(a)

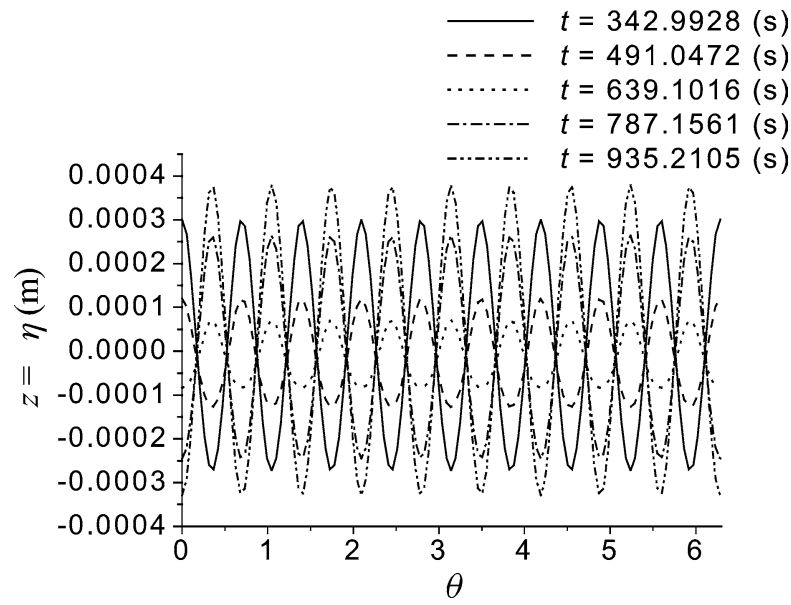

(b)

Fig. 12. Spatial evolution of surface wave displacement with radius $r$ and azimuth $\theta$ at different time (depth of fluid $h=1.0 \mathrm{~cm}$, radius of the vessel $R=7.5 \mathrm{~cm}$, forced frequency $f=29.01 \mathrm{~Hz}$, forced amplitude $A=11.4 \mu \mathrm{m}$ ). (a) Evolution of surface wave displacement with radius $r$ $(\theta=4 \pi / 3)$, (b) evolution of surface wave displacement with azimuth $\theta(r=0.5)$.

then (4.1) becomes following amplitude equation

$$
\mathrm{i} \frac{\mathrm{d} q(\tau)}{\mathrm{d} \tau}=-\mathrm{i} \beta_{1} q(\tau)+\sigma q(\tau)+M_{1} q^{2}(\tau) \bar{q}(\tau)+M_{2} \bar{q}(\tau) .
$$

It can be proved from (4.2) that the stable property of the amplitude $p(\tau)$ and $q(\tau)$ is equivalent, that is to say, if Eq. (4.3) is stable, then Eq. (4.1) is still stable, and vice versa. Divide the unknown variable into real and imaginary parts, and the amplitude equation (4.3) yields the following nonlinear ordinary differential equation groups

$$
\begin{aligned}
& \frac{\mathrm{d} q_{1}(\tau)}{\mathrm{d} \tau}=-\beta_{1} q_{1}(\tau)+\left(\sigma-M_{2}\right) q_{2}(\tau)+M_{1} q_{2}(\tau)\left[q_{1}^{2}(\tau)+q_{2}^{2}(\tau)\right], \\
& \frac{\mathrm{d} q_{2}(\tau)}{\mathrm{d} \tau}=-\beta_{1} q_{2}(\tau)-\left(\sigma+M_{2}\right) q_{1}(\tau)-M_{1} q_{1}(\tau)\left[q_{1}^{2}(\tau)+q_{2}^{2}(\tau)\right],
\end{aligned}
$$

where $q_{1}(\tau)$ and $q_{2}(\tau)$ are the real and imaginary parts of $q(\tau)$ respectively. In the following we will investigate the stability of linearized equation from (4.4) and (4.5). 


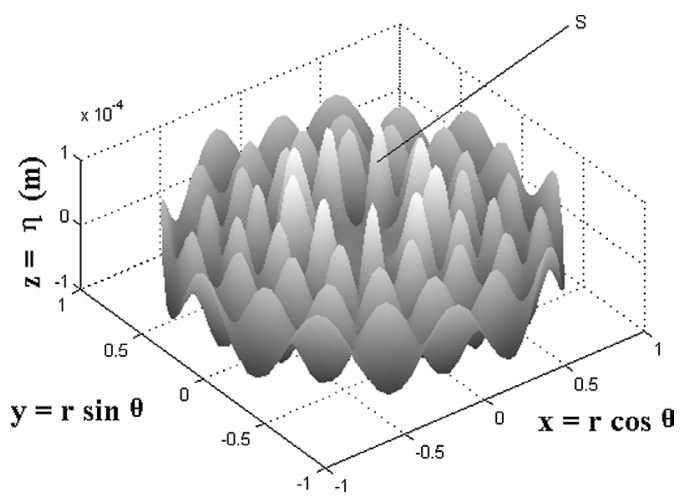

(a)

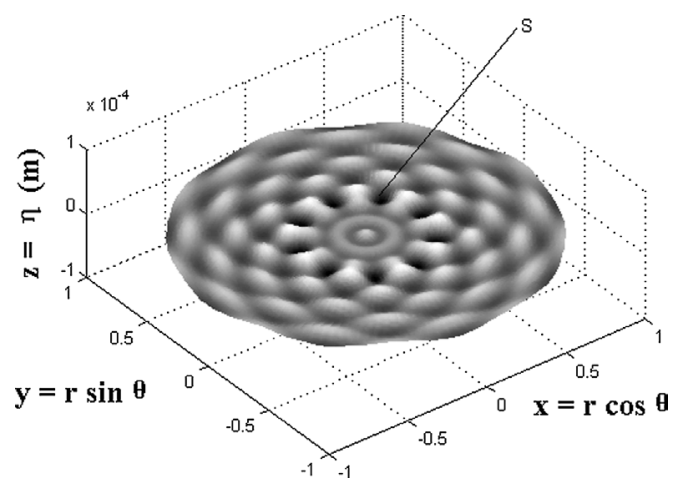

(c)

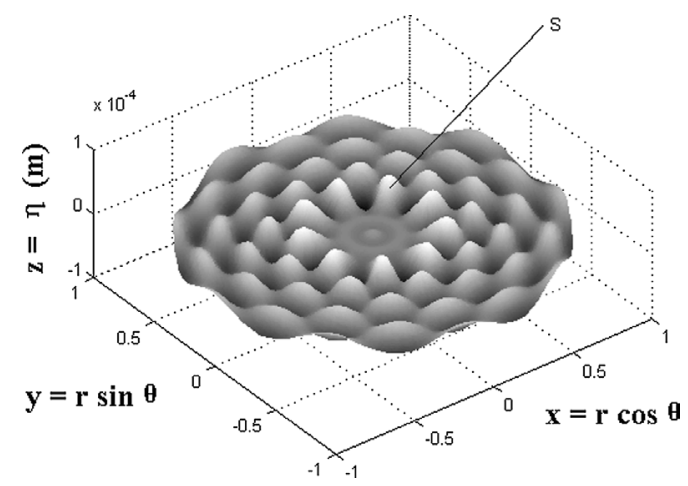

(b)

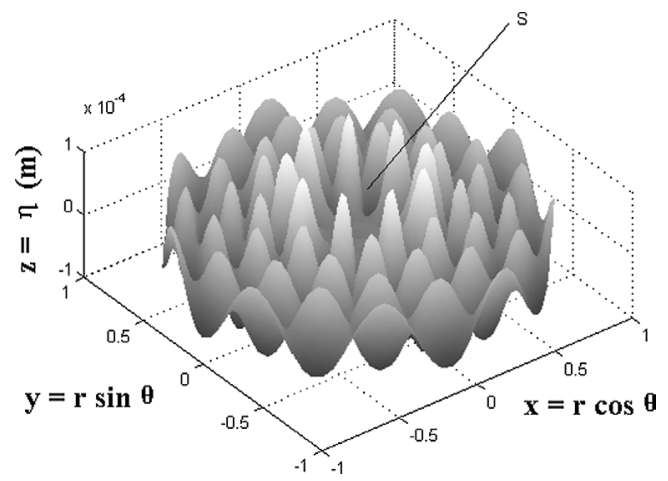

(d)

Fig. 13. Three-dimensional surface of free surface displacement at different time $t$ (depth of fluid $h=1.0 \mathrm{~cm}$, radius of the vessel $R=7.5 \mathrm{~cm}$, forced frequency $f=29.01 \mathrm{~Hz}$, forced amplitude $A=11.4 \mu \mathrm{m}$ ). (a) $t=0.563 \mathrm{~s}$, (b) $t=0.816 \mathrm{~s}$, (c) $t=0.872 \mathrm{~s}$, (d) $t=1.153 \mathrm{~s}$.

\subsection{The stability of the linearized equation}

In our parametrically excited problem, the unstable condition of linearized equation means the condition in which the surface wave can be formed. Neglect the nonlinear terms on the right of (4.4) and (4.5), and make $\hat{q}_{1}(\tau)$ and $\hat{q}_{2}(\tau)$ the infinitesimal disturbances associated to the zero solutions of $q_{1}(\tau)$ and $q_{2}(\tau)$. The disturbed equations $\hat{q}_{1}(\tau)$ and $\hat{q}_{2}(\tau)$ satisfy

$$
\begin{aligned}
& \frac{\mathrm{d} \hat{q}_{1}(\tau)}{\mathrm{d} \tau}=-\beta_{1} \hat{q}_{1}(\tau)+\left(\sigma-M_{2}\right) \hat{q}_{2}(\tau), \\
& \frac{\mathrm{d} \hat{q}_{2}(\tau)}{\mathrm{d} \tau}=-\left(\sigma+M_{2}\right) \hat{q}_{1}(\tau)-\beta_{1} \hat{q}_{2}(\tau) .
\end{aligned}
$$

The eigenfunction of (4.6) and (4.7) can be given as

$$
\left(\delta+\beta_{1}\right)^{2}=M_{2}^{2}-\sigma^{2} \text {. }
$$

Instability will happen if the real part of the eigenvalue $\delta$ is larger than zero, namely when

$$
M_{2}^{2}>\sigma^{2}+\beta_{1}^{2}
$$

the surface wave appears at the free surface. However, the free surface keeps plane if the real part of the eigenvalue $\delta$ is smaller than zero. Under this condition, the eigenvalue yields

$$
M_{2}^{2}<\sigma^{2}, \quad \text { or } \quad \sigma^{2}<M_{2}^{2}<\sigma^{2}+\beta_{1}^{2} \text {. }
$$

From the physical point of view, conditions (4.9) and (4.10) indicate that when the external forced energy is larger than that of the viscous dissipation, the surface wave appears due to the instability of the free surface. On the contrary, when the external forced energy is smaller than that of the viscous dissipation, the surface wave can not be produced.

Fig. 14 plots the results of instability, and the unstable regions are determined by (4.9). The shadow regions in Fig. 14 are the unstable regions. When the parameters enter these regions, the surface wave can be excited due to first instability. 


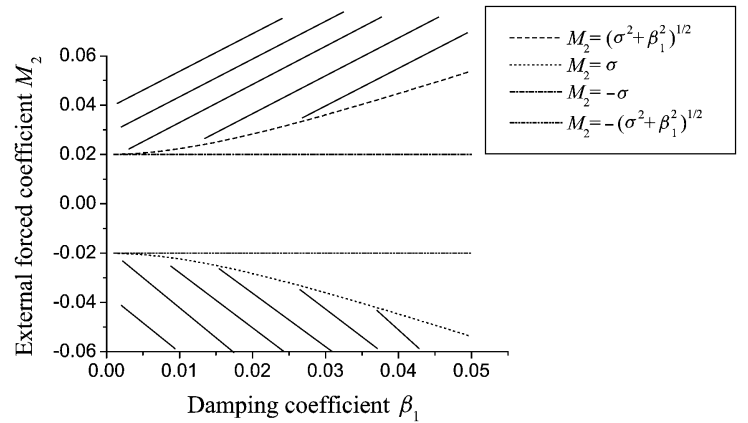

(a)

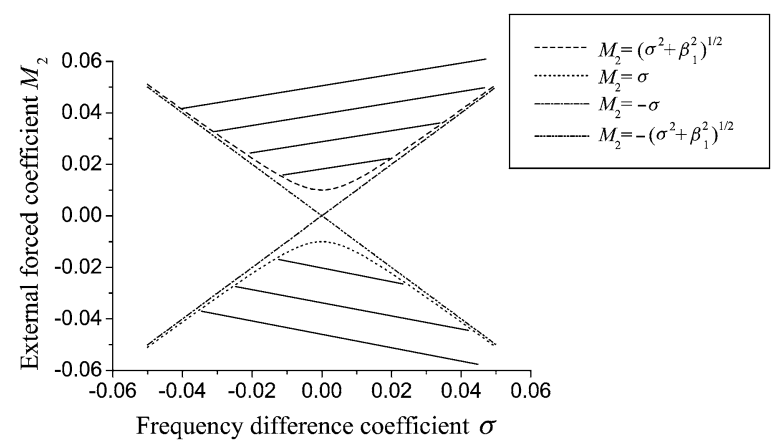

(b)

Fig. 14. Unstable region determined by Eq. (4.9). (a) Unstable region determined by damping coefficient $\beta_{1}$ and excited coefficient $M_{2}$ $(\sigma=0.02)$. (b) Unstable region determined by frequency difference coefficient $\sigma$ and excited coefficient $\left(\beta_{1}=0.01\right)$.

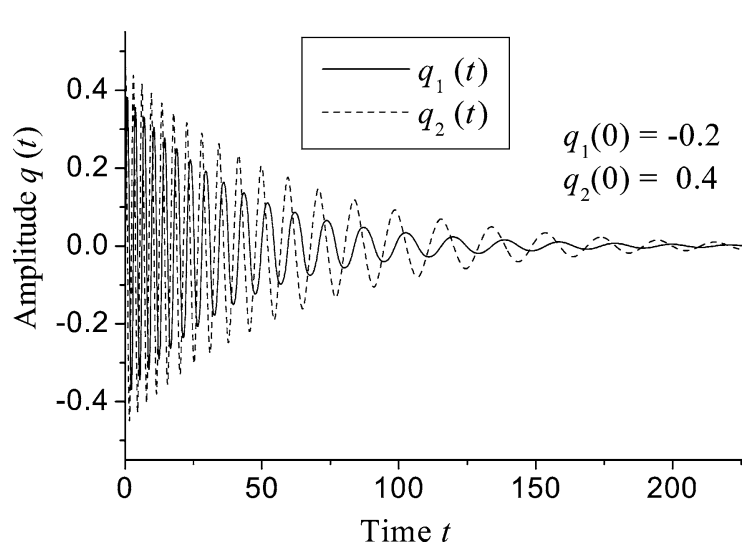

(a)

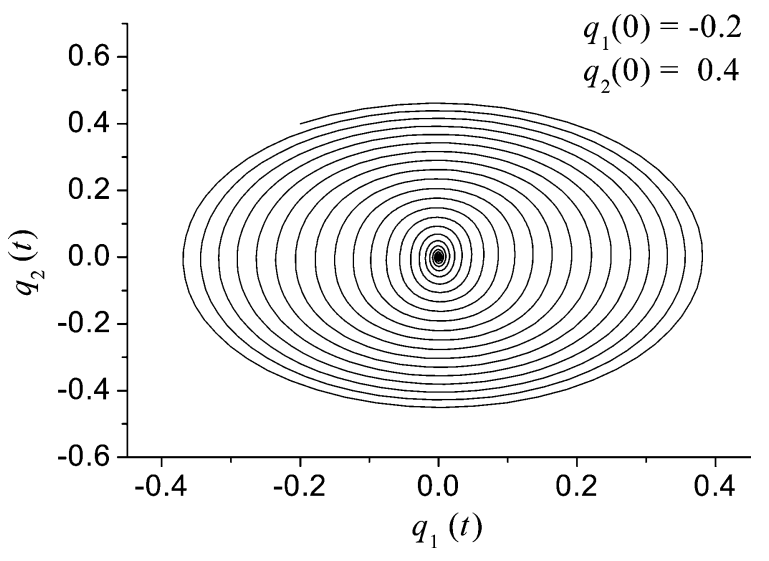

(b)

Fig. 15. The evolution of the amplitude $q(\tau)$ with time and phase-plane trajectory $\left(\sigma=0.5, M_{1}=10, M_{2}=0.4, \beta_{1}=0.02\right)$. (a) The evolution of the amplitude $q(\tau)$ with time, (b) the phase-plane trajectory of $q_{1}(\tau)$ and $q_{2}(\tau)$.

\subsection{The stability of the nonlinear amplitude equation}

Physically, the stability analysis in this subsection means "second instability". That is to say, for a prescribed stable surface wave, in which kind of condition the surface wave is stable or unstable. In Eq. (4.3), let the differential related to the time equal to zero, the nonzero equilibrium solution yields

$$
\mathrm{i} \beta_{1} q(\tau)=\sigma q(\tau)+M_{1} q^{2}(\tau) \bar{q}(\tau)+M_{2} \bar{q}(\tau) .
$$

Let $q_{0}=a_{0} \mathrm{e}^{\mathrm{i} \vartheta} \neq 0$ ( $a_{0}$ is a real number) is an equilibrium solution of (4.11), we have

$$
a_{0}=\left[\frac{-\sigma \pm \sqrt{M_{2}^{2}-\beta_{1}^{2}}}{M_{1}}\right]^{1 / 2}, \quad \sin 2 \vartheta=-\frac{\beta_{1}}{M_{2}} .
$$

Supposing $q^{\prime}(\tau)$ is an infinitesimal disturbance associated to the equilibrium solution $q_{0}$, taking disturbed expression $q_{1}(\tau)=$ $q^{\prime}(\tau)+q_{0}$ into (4.11), and ignoring the nonlinear term of infinitesimal disturbance, the disturbed equation can be written as

$$
\mathrm{i} \frac{\mathrm{d} q^{\prime}(\tau)}{\mathrm{d} \tau}=-\mathrm{i} \beta_{1} q^{\prime}(\tau)+\sigma q^{\prime}(\tau)+M_{2} \bar{q}^{\prime}(\tau)+M_{1}\left(q_{0}^{2} \bar{q}^{\prime}(\tau)+2\left|q_{0}\right|^{2} q^{\prime}(\tau)\right) .
$$

Separate (4.13) into real and imaginary parts, let $q^{\prime}(\tau)=A_{1}(\tau)+\mathrm{i} A_{2}(\tau)$, we have the following ordinary differential equation group

$$
\frac{\mathrm{d} A_{1}}{\mathrm{~d} \tau}=-\beta_{1}\left[1+\frac{-\sigma \pm \sqrt{M_{2}^{2}-\beta_{1}^{2}}}{M_{2}}\right] A_{1}+\left[\left(\sigma-M_{2}\right)+\left(-\sigma \pm \sqrt{M_{2}^{2}-\beta_{1}^{2}}\right)\left(2 \pm \frac{\sqrt{M_{2}^{2}-\beta_{1}^{2}}}{M_{2}}\right)\right] A_{2},
$$




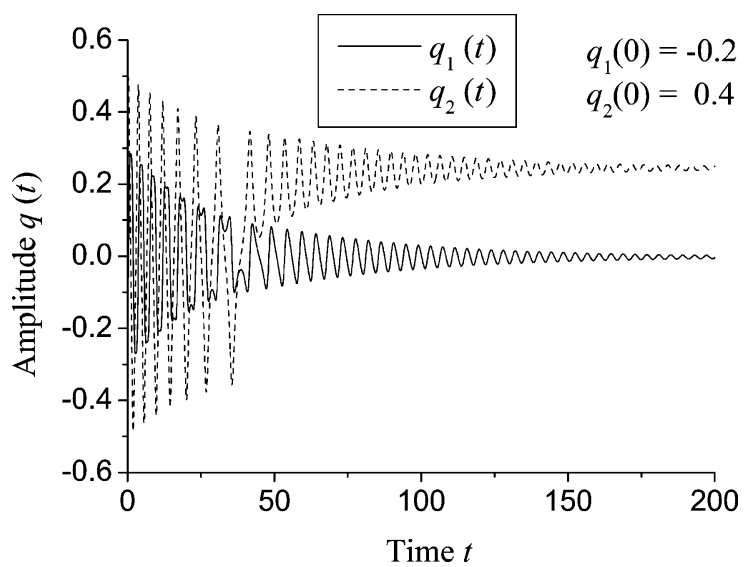

(a)

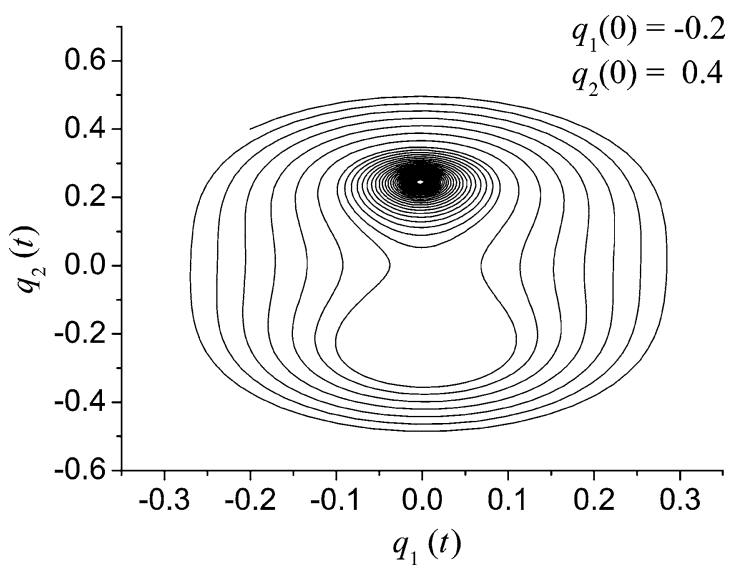

(b)

Fig. 16. The evolution of the amplitude $q(\tau)$ with time and phase-plane trajectory $\left(\sigma=0.4, M_{1}=10, M_{2}=1, \beta_{1}=0.02\right)$. (a) The evolution of the amplitude $q(\tau)$ with time, (b) the phase-plane trajectory of $q_{1}(\tau)$ and $q_{2}(\tau)$.

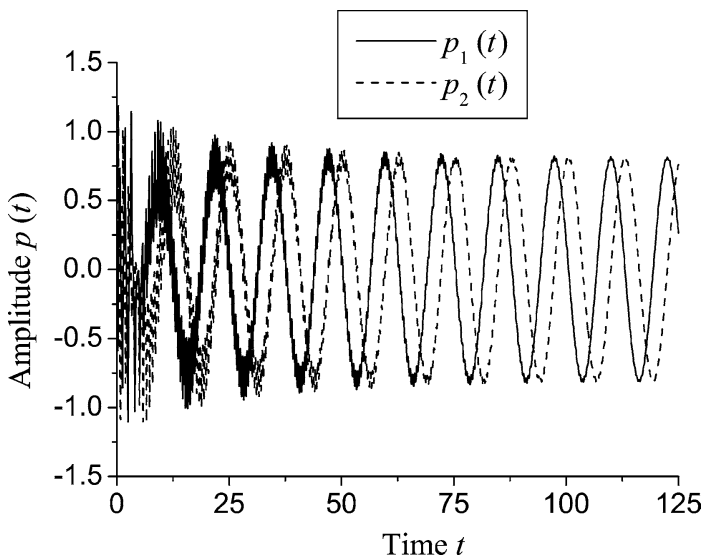

(a)

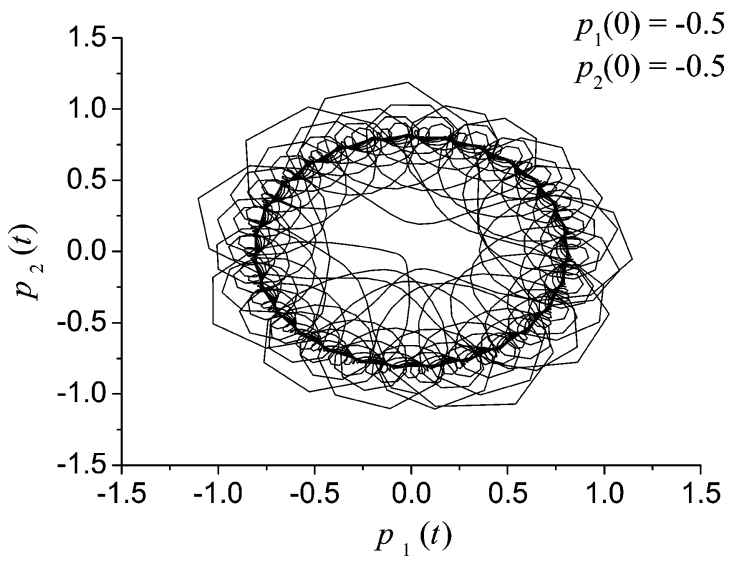

(b)

Fig. 17. The evolution of the amplitude $p(\tau)$ with time and phase-plane trajectory $\left(\sigma=0.4, M_{1}=10, M_{2}=1, \beta_{1}=0.02\right)$. (a) The evolution of the amplitude $p(\tau)$ with time, (b) the phase-plane trajectory of $p_{1}(\tau)$ and $p_{2}(\tau)$.

$$
\frac{\mathrm{d} A_{2}}{\mathrm{~d} \tau}=\left[-\left(\sigma+M_{2}\right)-\left(-\sigma \pm \sqrt{M_{2}^{2}-\beta_{1}^{2}}\right)\left(2 \mp \frac{\sqrt{M_{2}^{2}-\beta_{1}^{2}}}{M_{2}}\right)\right] A_{1}-\beta_{1}\left[1-\frac{-\sigma \pm \sqrt{M_{2}^{2}-\beta_{1}^{2}}}{M_{2}}\right] A_{2} .
$$

The eigenfunction of (4.14) and (4.15) can be given as

$$
\left(\delta+\beta_{1}\right)^{2}=-4\left[M_{2}^{2}-\frac{5}{4} \beta_{1}^{2} \mp \sigma \sqrt{M_{2}^{2}-\beta_{1}^{2}}\right] .
$$

By analysis to Eq. (4.16), whenever $M_{1}>0$ or $M_{1}<0$, if the condition $M_{2}^{2}>\sigma^{2}+\beta_{1}^{2}$ is fulfilled, stable surface waves can be formed. It can also be shown that the equilibrium mode will not lose its stability to an infinitesimal disturbance by method of contradiction.

\subsection{Properties of the modified nonlinear amplitude equation}

In this subsection, we carry out some numerical computations for the modified amplitude equation group (4.4) and (4.5) by fourth order Runge-Kutta approach with equivalent time-step. The evolution laws of the amplitudes with time are investigated in different parametrical regions. The properties of the amplitude are presented by phase-plane trajectory. The evolutions of the amplitude with time and the phase-plane trajectory are depicted in Fig. 15. The choice of the parameters satisfies the first condition of (4.10). (In Figs. 15-17, all the time ' $t$ ' in both horizontal and vertical coordinates means the ' $\tau$ ' corresponding to Eqs. (4.4) and (4.5).) 

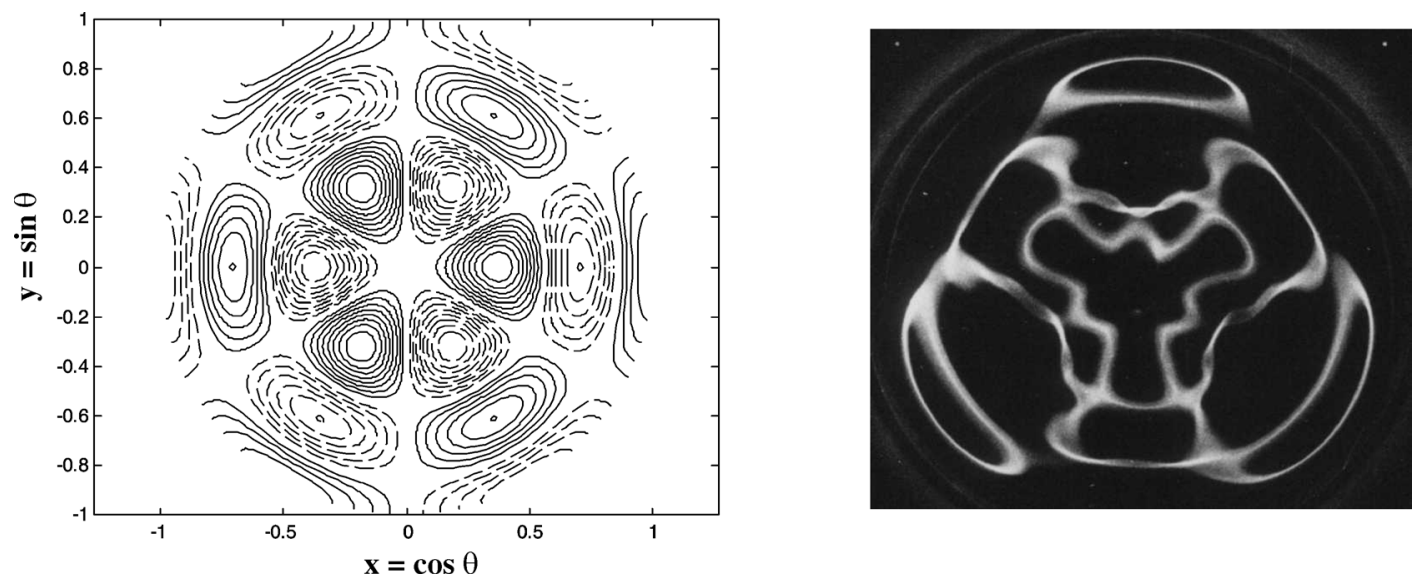

(a)
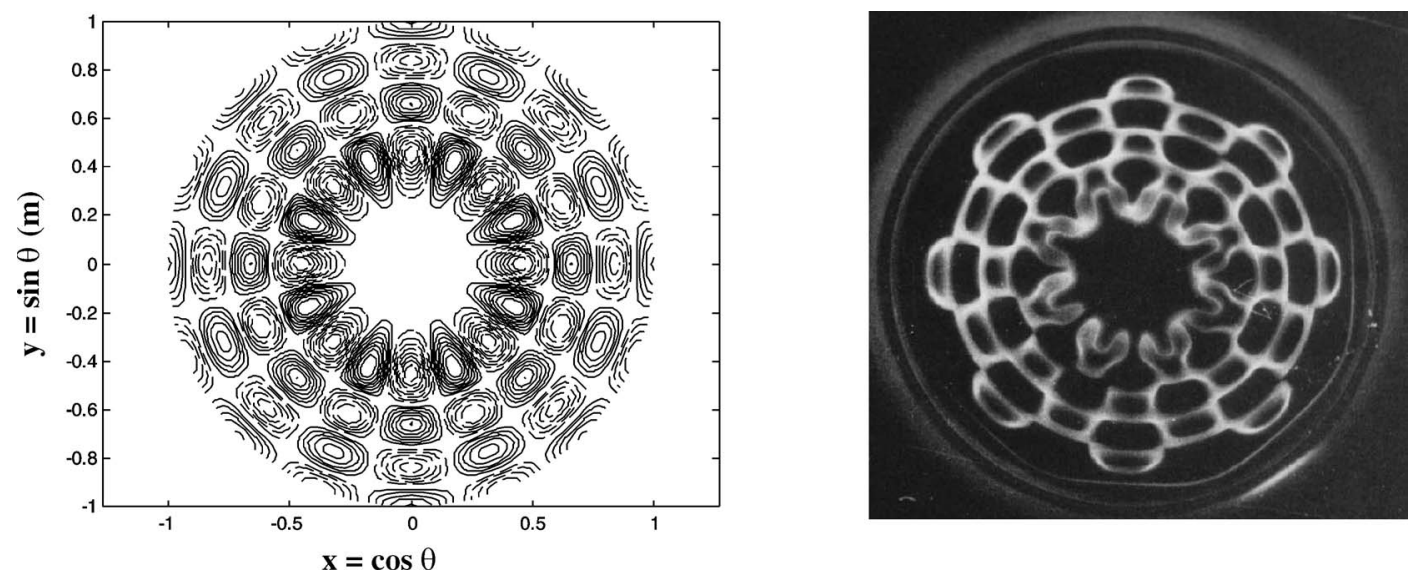

(b)
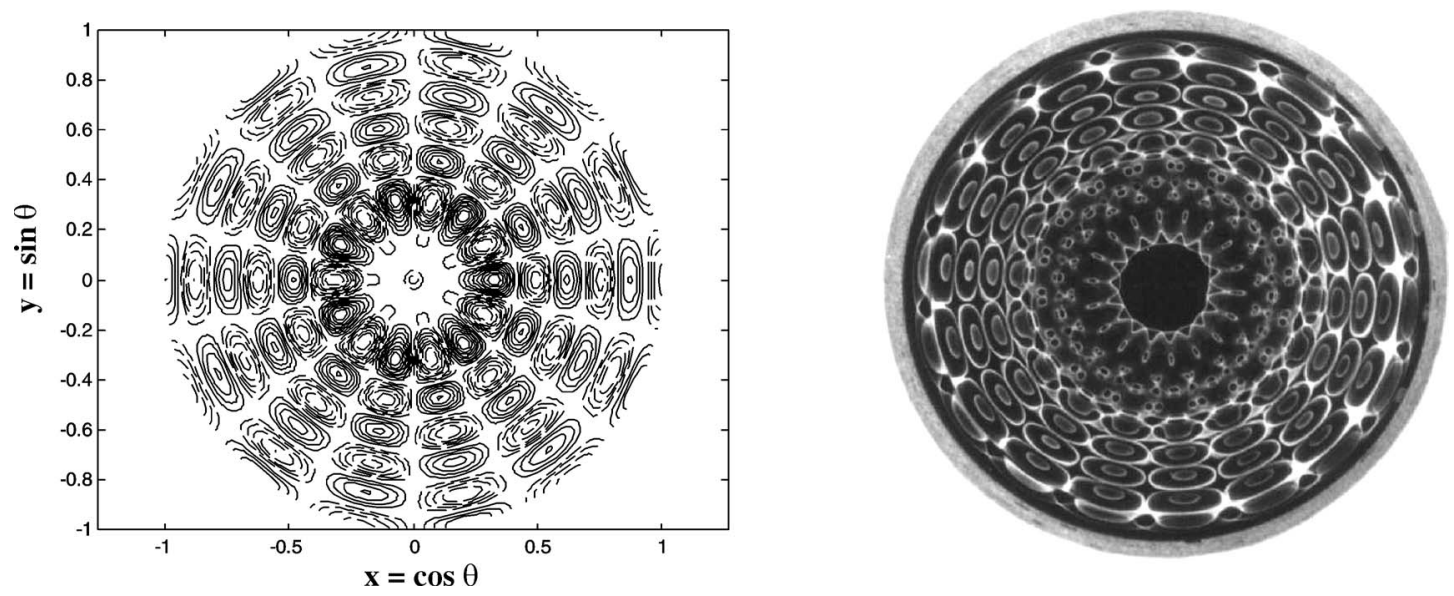

(c)

Fig. 18. Comparison of theoretical contours of the surface wave patterns with those of experiment (depth of fluid $h=1.0 \mathrm{~cm}$, radius of the vessel $R=7.5 \mathrm{~cm}$, surface tension $\Gamma=0.072716 \mathrm{~N} / \mathrm{m}$, viscosity coefficient $v=10^{-6} \mathrm{~m}^{2} / \mathrm{s}$ ). (a) $(3,3)$ mode, (left) forced frequency $19.9 \mathrm{~Hz}$, (right) forced frequency $20 \mathrm{~Hz}$, (b) $(8,4)$ mode, (left) forced frequency $22.5 \mathrm{~Hz}$, (right) forced frequency $50 \mathrm{~Hz}$, (c) $(9$, 5) mode, (left) forced frequency $26.5 \mathrm{~Hz}$, (right) forced frequency $52 \mathrm{~Hz}$. 
It can be easily seen from Fig. 15 that the amplitude decreases gradually and tends to zero eventually under the prescribed initial conditions. This indicates that the external driven energy can not overcome the viscous dissipation, and the stable surface wave can not be formed.

However, when the parameters yield the condition (4.9), the instability will happen and the surface wave will appear. Fig. 16 illustrated the evolution of the amplitude with time and corresponding phase-plane trajectory. It can be seen from figure 16 that the amplitude tends to a constant with the development of the time, and the stable surface wave can be formed.

Fig. 17 illustrated the development of the primitive amplitude $p(\tau)$ with time, which corresponds to transformed amplitude $q(\tau)$ in Fig. 16. Fig. 17(a) shows the surface wave is oscillating at first and a stable periodical solution can be formed with the development of the time. Similarly, Fig. 17(b) indicates that the surface wave tends to a stable limit cycle with the evolution of the time.

\subsection{The comparison of experimental flowing patterns with those in theory}

We have studied the pattern-forming both in inviscid fluid with the effect of surface tension and in weakly viscous fluid for the vertically excited system of the circular cylindrical vessel. The modification was made by adding the viscous damping to the natural frequency of the surface wave. In Fig. 18, a comparison of theoretical contours of the surface wave can be made with those in experiment at different forced frequency (forced amplitude is 11.4 micron). It can be found from Fig. 18 that when the wave number is small, such as $(3,3)$ mode, the computational forced frequency agrees well with that of the experiment. However, when the wave number is large, such as $(8,4)$ and $(9,5)$ modes, the discrepancy of forced frequency is large. The reason maybe caused by moving contact line effect between side-wall and surface of fluid, wetting phenomenon, and mode competition, etc., which are ignored in this paper.

\section{Conclusions}

Both in the inviscid and weakly viscous fluids, we examined the vertically excited surface waves in a circular cylindrical vessel by two-time scale perturbation expansions. We found the following results:

1. The structures of the surface waves become complex with the increase of the forced frequency. Many unreported surface wave modes were found and standing wave character was confirmed.

2. The analytical expression of the damping coefficient was obtained and its properties were studied over a range of system parameters. The viscous damping increases with viscosity, depth of the fluid and decreases with external amplitude or frequency excitation. In addition, a comparison of the damping derived from our present theory with that of Case and Parkinson [27] was made, and the results are qualitatively accordance with each other.

3. When the wave number is small, the influence of the damping is important. However, when the wave number is large, the effect of the surface tension is prominent.

4. A necessary condition to insure the appearance of the surface wave is obtained by the instability investigation of the amplitude equation. The equilibrium surface wave will not lose its stability to an infinitesimal disturbance.

\section{Acknowledgements}

Referees of an original version have been most helpful in their criticisms. The authors are grateful to Prof. Zhou Xian-chu for discussion on the aspects of mathematical treatment, and Dr. Bai Wei for assistance with the numerical work. This research project is supported by the National Natural Science Foundation of China (Grant No: 19772063, 19772068).

\section{References}

[1] T.B. Epperson, R. Brown, H.N. Abramson, Dynamic loads resulting from fuel motion in missile tanks, in: Advances in Ballistic Missile and Space Technology, vol. 2, Pergamon, New York, 1961, pp. 313-327.

[2] H.N. Abramson, Dynamic behavior of liquid in moving container, Appl. Mech. Rev. 16 (7) (1963) 501-506.

[3] M. Faraday, On the forms and states assumed by fluids in contact with vibrating elastic surfaces, Philos. Trans. Roy. Soc. London 121 (1831) 319-340.

[4] J.W. Miles, D. Henderson, Parametrically forced surface waves, Ann. Rev. Fluid Mech. 22 (1990) 419-448.

[5] X.C. Zhou, Forced soliton in fluid mechanics, Adv. in Mech. 8 (3) (1998) 374-382 (in Chinese).

[6] S. Ciliberto, J.P. Gollub, Phenomenological model of chaotic mode competition in surface wave, Nuovo Cimento D6 (1985) $309-316$. 
[7] E. Meron, I. Procaccia, Low dimensional chaos in surface waves: theoretical analysis of an experiment, Phys. Rev. A 34 (1986) $3221-3237$.

[8] Y.L. Cheng, Advances in studies of internal solitary waves in stratified fluids: a review, Adv. in Mech. 28 (3) (1998) 383-391 (in Chinese).

[9] T.B. Benjamin, F. Ursell, The stability of the plane free surface of a liquid in vertical periodic motion, Proc. Roy. Soc. London 255 (1954) $505-515$

[10] J.W. Miles, Nonlinear surface waves in closed basins, J. Fluid Mech. 75 (1976) 419-448.

[11] J.W. Miles, Parametrically excited solitary waves, J. Fluid Mech. 148 (1984) 451-460.

[12] J.W. Miles, On Faraday waves, J. Fluid Mech. 248 (1993) 671-683.

[13] S. Douady, Experimental study of the Faraday instability, J. Fluid Mech. 221 (1990) 383-409.

[14] W.S. Edwards, S. Fauve, Patterns and quasi-patterns in the Faraday experiment, J. Fluid Mech. 278 (1994) 123-148.

[15] M. Nagata, Nonlinear Faraday resonance in a box with a square base, J. Fluid Mech. 209 (1989) 265-284.

[16] P.J. Holmes, Chaotic motions in a weakly nonlinear model for surface waves, J. Fluid Mech. 162 (1986) 365-388.

[17] S. Ciliberto, J.P. Gollub, Pattern competition leads to chaos, Phys. Rev. Lett. 52 (1984) 922-925.

[18] A. Kudrolli, J.P. Gollub, Pattern and spatiotemporal chaos in parametrically forced surface waves: a systematic survey at large aspect ratio, Physica D 97 (1996) 113-154.

[19] X.Q. E, Y.X. Gao, Ordered and chaotic modes of surface wave patterns in a vertically oscillating fluid, Commun. Nonlinear Sci. Numer. Simul. 1 (2) (1996) 1-5.

[20] X.Q. E, Y.X. Gao, Visualization of surface wave patterns of a fluid in vertical vibration, in: Proceedings of the Fourth Asian Symposium on Visualization, Beijing, 1996, pp. 653-658.

[21] Y.X. Gao, X.Q. E, Surface wave visualization for liquid in micron-amplitude vibration, J. Experim. Mech. 13 (3) (1998) $326-333$ (in Chinese).

[22] Y.J. Jian, X.Q. E, W. Bai, Nonlinear Faraday waves in a parametrically excited circular cylindrical container, Appl. Math. Mech. 24 (10) (2003) 1194-1207. English Edition.

[23] Y.J. Jian, X.Q. E, Surface wave structure in a vertically forced circular cylindrical vessel, J. Hydrodynamics A 18 (2) (2003) 135-147 (in Chinese).

[24] F. Ursell, Edge waves on a sloping beach, Proc. Roy. Soc. London Ser. A 214 (1952) 79-97.

[25] C.C. Mei, L.F. Liu, The damping of surface gravity waves in a bounded liquid, J. Fluid Mech. 59 (2) (1973) $239-256$.

[26] A.J. Bernoff, L.P. Kwok, S. Lichter, Viscous cross-waves: an analytical treatment, Phys. Fluids A 1 (1989) 678-688.

[27] K.M. Case, W.C. Parkinson, Damping of surface waves in an incompressible liquid, J. Fluid Mech. 2 (1957) 172-184.

[28] D.M. Henderson, J.W. Miles, Surface-wave damping in a circular cylinder with a fixed contact line, J. Fluid Mech. 275 (1994) $285-299$.

[29] C. Martel, J.A. Nicolás, J.M. Vega, Surface-wave damping in a brimful circular cylinder, J. Fluid Mech. 360 (1998) $213-228$.

[30] T.S. Krasnopolskays, G.J.F. van Heijst, Wave pattern formation in a fluid annulus with a radially vibrating inner cylinder, J. Fluid Mech. 328 (1996) 229-252.

[31] G.H. Keulegan, Energy dissipation in standing waves in rectangular basins, J. Fluid Mech. 6 (1959) 33-50.

[32] D.M. Henderson, Effects of surfactants on Faraday-wave dynamics, J. Fluid Mech. 365 (1998) 89-107. 